\title{
VEGF is a modifier of amyotrophic lateral sclerosis in mice and humans and protects motoneurons against ischemic death
}

\author{
Diether Lambrechts ${ }^{1}$, Erik Storkebaum ${ }^{1}$, Masafumi Morimoto ${ }^{1}$, Jurgen Del-Favero ${ }^{2}$, Frederik Desmet ${ }^{1}$, \\ Stefan L Marklund ${ }^{3}$, Sabine Wyns ${ }^{1}$, Vincent Thijs ${ }^{1}$, Jörgen Andersson ${ }^{3}$, Ingrid van Marion ${ }^{4}$, Ammar Al-Chalabi ${ }^{5}$, \\ Stephanie Bornes ${ }^{6}$, Rhiannon Musson ${ }^{4}$, Valerie Hansen ${ }^{5}$, Lars Beckman ${ }^{3}$, Rolf Adolfsson ${ }^{7}$, Hardev Singh Pall ${ }^{4}$, \\ Hervé Prats ${ }^{6}$, Severine Vermeire ${ }^{1}$, Paul Rutgeerts ${ }^{1}$, Shigehiro Katayama ${ }^{8}$, Takuya Awata ${ }^{8}$, Nigel Leigh ${ }^{5}$, Loïc Lang- \\ Lazdunski $^{9}$, Mieke Dewerchin ${ }^{1}$, Christopher Shaw ${ }^{5}$, Lieve Moons ${ }^{1}$, Robert Vlietinck ${ }^{1,10}$, Karen E Morrison ${ }^{4}$, \\ Wim Robberecht ${ }^{1}$, Christine Van Broeckhoven $^{2}$, Désiré Collen ${ }^{1}$, Peter M Andersen ${ }^{3} \&$ Peter Carmeliet $^{1}$
}

Amyotrophic lateral sclerosis (ALS) is an incurable degenerative disorder of motoneurons. We recently reported that reduced expression of Vegfa causes ALS-like motoneuron degeneration in Vegfa ${ }^{\partial / \partial}$ mice. In a meta-analysis of over 900 individuals from Sweden and over 1,000 individuals from Belgium and England, we now report that subjects homozygous with respect to the haplotypes $-2,578 \mathrm{~A} /-1,154 \mathrm{~A} /-634 \mathrm{G}$ or $-2,578 \mathrm{~A} /-1,154 \mathrm{G} /-634 \mathrm{G}$ in the $V E G F$ promoter/leader sequence had a 1.8 times greater risk of ALS $(P=\mathbf{0 . 0 0 0 0 4})$. These 'at-risk' haplotypes lowered circulating VEGF levels in vivo and reduced $V E G F$ gene transcription, IRES-mediated VEGF expression and translation of a novel large-VEGF isoform (L-VEGF) in vivo. Moreover,

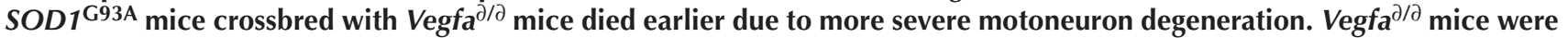
unusually susceptible to persistent paralysis after spinal cord ischemia, and treatment with Vegfa protected mice against ischemic motoneuron death. These findings indicate that VEGF is a modifier of motoneuron degeneration in human ALS and unveil a therapeutic potential of Vegfa for stressed motoneurons in mice.

ALS, also called Lou Gehrig or Charcot disease, is a devastating untreatable neurodegenerative disorder with a yearly incidence of $1-2$ per 100,000 (refs. $1-5$ ). It is characterized by progressive wasting and weakness of limb, bulbar and respiratory muscles due to degeneration of motoneurons in the spinal cord, brain stem and motor cortex. Disease onset is usually in the fifth or sixth decade of life and, in most affected individuals, progresses to death due to respiratory failure 3-5 years after onset. There are unexplained large individual differences in clinical presentation (initial symptoms may be spinal or bulbar in nature), age of onset (ranging from juvenile to the very elderly) and survival time (from a few months to more than 20 years). Elucidating the modifying factors has important consequences for our understanding of neuron degeneration and development of therapy.
Approximately $5-10 \%$ of ALS is familial ${ }^{6,7}$. Five types of familial ALS (FALS) have been assigned to loci of the human genome ${ }^{6,8}$. ALS1, an autosomal dominant form of adult ALS affecting 14-23\% of individuals with FALS, is associated with more than 100 mutations in the gene encoding $\mathrm{Cu} / \mathrm{Zn}$ superoxide dismutase (SOD1) on chromosome 21q22.1-2. Recently, the gene associated with ALS2, an autosomal recessive form of juvenile ALS, was reported to encode a protein containing domains with homology to Ran/Rho-GEFs ${ }^{9}$. Dysfunction of dynactin 1-mediated axonal transport can also lead to human motoneuron disease. The genetic causes of the other forms of FALS are not yet known. The etiology and pathogenesis of the more common sporadic form of the disease are poorly understood $^{1-4,10}$. Besides a family history of ALS, age and male gender are the only established risk factors for ALS. Sporadic ALS (SALS) is

${ }^{1}$ The Center for Transgene Technology and Gene Therapy, Flanders Interuniversity Institute for Biotechnology and Departments of Neurology and Internal Medicine, University Hospital Gasthuisberg, KU Leuven, Leuven, B-3000, Belgium. ${ }^{2}$ Department of Molecular Genetics, Flanders Interuniversity Institute for Biotechnology, University of Antwerp, B-2610 Antwerpen, Belgium. ${ }^{3}$ Department of Neurology, Umea University Hospital, and Institute of Clinical Neuroscience and Medical Genetics, Umea University, S-901 85 Umeå, Sweden. ${ }^{4}$ Department of Neurology, The Medical School, University of Birmingham, Edgbaston, B15 2TT, UK. ${ }^{5}$ Departments of Neurology and Medical and Molecular Genetics, Guy's, King's and St. Thomas' School of Medicine and Institute of Psychiatry, King's College London, London SE5 8AF, UK. 6 INSERM 397, Endocrinologie et Communication Cellulaire, Institut Fédératif de Recherche Louis Bugnard, CHU Rangueil, 31403 Toulouse, CEDEX 4, France. ${ }^{7}$ Department of Clinical Sciences, Division of Psychiatry, Umea University, Umea, Sweden. ${ }^{84 t h}$ Department of Medicine, Saitama Medical School, Moroyama, Iruma-gun, Saitama 350-0495, Japan. 99Service de Chirurgie Thoracique, Hôpital Européen Georges Pompidou, 75015 Paris, France.

${ }^{10}$ Department of Population Genetics, Genomics \& Bioinformatics, University Maastricht, 6200 MD Maastricht, The Netherlands. Correspondence should be addressed to P.C. (peter.carmeliet@med.kuleuven.ac.be). 
believed to be a multi-factorial disease in which modifying genes and environmental agents affect its clinical manifestation, but few associated genes have so far been identified ${ }^{7,11-16}$.

Using Cre/loxP-mediated gene targeting to delete the hypoxiaresponse element (HRE) in the promoter region of the gene encoding vascular endothelial growth factor in mice ( Vegfa ${ }^{\operatorname{tm} 2 \mathrm{Pec}}$, herein called $V e g f a^{\partial}$ ), we recently showed that impaired expression of Vegfa predisposed $V e g f a / \partial$ mice to adult-onset progressive motoneuron degeneration, with many neuropathological and clinical signs reminiscent of human ALS ${ }^{17,18}$. It was not clear, however, whether VEGF is also involved in the pathogenesis of human ALS. Here, we show that $V E G F$ is a modifier gene associated with motoneuron degeneration in individuals with ALS and in a mouse model of ALS. In addition, we provide evidence that treatment of mice with Vegfa stimulates motoneuron survival in stressed conditions.

\section{RESULTS}

Analysis of the HRE, HIF1A and EPAS1 in individuals with ALS A targeted deletion of the HRE in the Vegfa promoter impaired hypoxic upregulation of the expression of Vegfa and caused adultonset motoneuron degeneration in Vegfa ${ }^{\partial / \partial}$ mice $^{17}$. We therefore analyzed whether variations in the HRE sequence confer greater risk for ALS in humans. We found no sequence alterations in individuals with SALS or FALS from Sweden $(n=250)$, Belgium $(n=96)$ or England $(n=94)$. Because hypoxic upregulation of VEGF occurs through binding of the HIF1A and EPAS1 (also called HIF2A) transcription complexes to the HRE region, we also screened 96 individuals with SALS and 200 unaffected individuals from Sweden for sequence variations in HIF1A and EPAS1. We did not detect any exonic sequence variations causing functionally relevant missense mutations in $H I F 1 A$ or EPAS1 (data not shown). We then examined whether singlenucleotide polymorphisms (SNPs) in VEGF might confer a greater risk for motoneuron degeneration in individuals with ALS. Because both baseline and hypoxia-induced Vegfa expression levels were reduced in $V e g f a^{\partial / \partial}$ mice $^{17}$, we tested whether SNPs downregulating expression of VEGF might increase the susceptibility to ALS. We analyzed variations in the promoter or $5^{\prime}$ untranslated region (UTR) of $V E G F$, which previously have been documented to downregulate VEGF expression: 2,578C $\rightarrow \mathrm{A}$ (ref. 19), 1,154G $\rightarrow \mathrm{A}$ (ref. 19) and $634 \mathrm{G} \rightarrow \mathrm{C}$ (ref. 20; Fig. 1a).

\section{VEGF variations and ALS in the Swedish population}

We initially genotyped 349 individuals with SALS and FALS and 275 unrelated healthy controls of similar age, geography and ethnicity drawn randomly from a population-based prospective study cohort from northern Sweden (Table 1). None of the genotype frequencies in the control population deviated from Hardy-Weinberg equilibrium (Table 2). We found no differences between affected individuals and controls in the distribution of the $-2,578 \mathrm{C} \rightarrow \mathrm{A}(P=0.41)$ or $-634 \mathrm{G} \rightarrow \mathrm{C}(P=0.61)$ variations, but the $-1,154 \mathrm{~A}$ allele was more prevalent in affected individuals than controls (allele frequency of 0.33 in affected individuals versus 0.28 in controls; $P=0.029$ ). Individuals
Figure 1 Genetic association of VEGF with ALS (a) Structure of VEGF. The SNPs in the promoter and 5' UTR, the ATG codon of the secreted VEGF isoforms (only VEGF ${ }^{189}$ is shown) and the CTG1 codon of L-VEGF are indicated. Cleavage of L-VEGF after the signal peptide (blue arrow) generates the secreted VEGF forms. (b) Median VEGF plasma levels in individuals with SALS and spouses from Sweden. Differences were calculated between the indicated genotypes and all others (Mann-Whitney U-test) in the spouses ( ${ }^{a} P=$ $\left.0.13,{ }^{b} P=0.17,{ }^{c} P=0.08\right)$ and in the affected individuals ( ${ }^{\mathrm{d}} P=0.016,{ }^{\mathrm{e}} P=0.008$, $\left.{ }^{\mathrm{f}} P=0.005\right)$. VEGF levels were compared between affected individuals and spouses of the indicated genotypes $\left({ }^{g} P<0.001,{ }^{\mathrm{h}} P=\right.$ $\left.0.26,{ }^{i} P=0.011,{ }^{j} P=0.008\right)$. (c,d) Models of the predicted two-dimensional structure of the 5' UTR containing the common -634G (c) or variant $-634 \mathrm{C}$ (d) allele. The structure of the $5^{\prime}$ leader sequence of both alleles is comparable, except in the core region of the IRES-B sequence upstream of the CUG1 start codon of L-VEGF, which is substantially remodeled. (e) Western blot of L-VEGF and VEGF ${ }^{189}$ in extracts of COS cells transfected with a VEGF expression cassette containing the entire $5^{\prime}$ UTR in-frame with the cDNA encoding the VEGF ${ }^{189}$ isoform. (f) Correlation of median VEGF serum values with the different genotypes in the Belgian control population. Statistical differences were calculated by comparing the indicated genotypes with all other genotypes (Mann-Whitney U-test; ${ }^{a} P=$

$0.02,{ }^{b} P=0.017,{ }^{c} P=0.0005$ ). (g) Meta-analysis of the ALS risk (ORs and $95 \%$ c.i.) associated with the AAG/AAG and AGG/AGG genotypes (combined) in individual and pooled populations. Breslow-Day homogeneity test showed no heterogeneity between the observed ORs in the different subpopulations $(P=0.32)$.
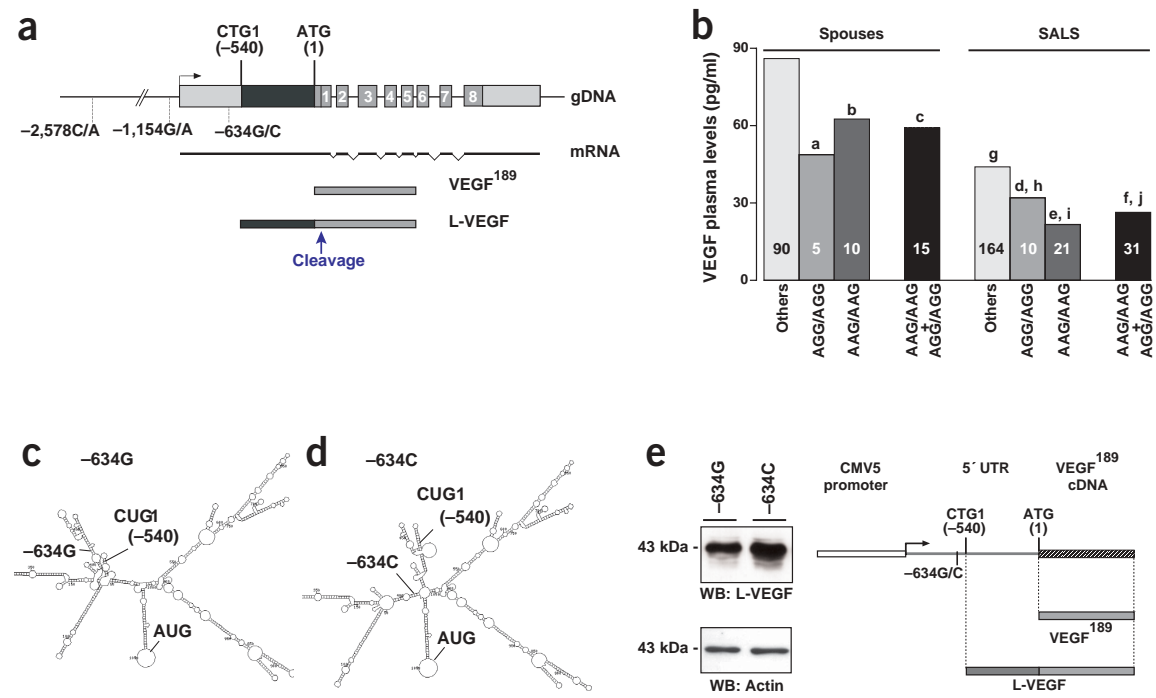

f

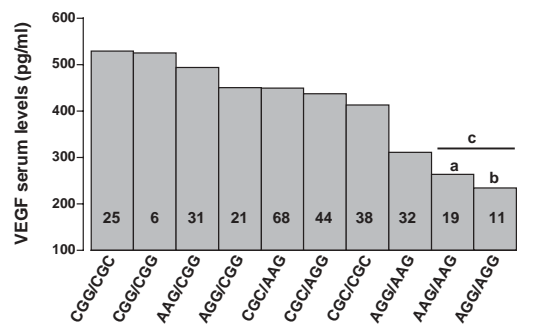

g

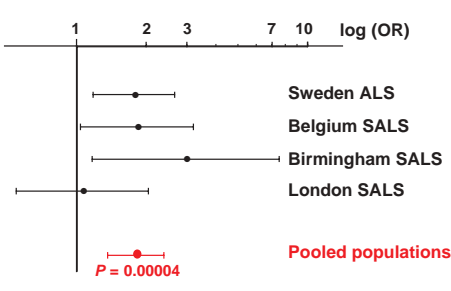


Table 1 Characteristics of study populations

\begin{tabular}{|c|c|c|c|c|c|c|}
\hline Study populations & Total & Male/female & Age at onset ${ }^{a}$ & Survival $^{\mathrm{b}}$ & Spinal onset & Bulbar onset \\
\hline \multicolumn{7}{|l|}{ Sweden } \\
\hline SALS & 292 & $165 / 127$ & $64(20-90)$ & c & 202 & 90 \\
\hline FALS & 57 & 29/28 & $62(10-81)$ & c & 45 & 12 \\
\hline Population-based controls & 275 & $138 / 137$ & $60(50-75)$ & - & - & - \\
\hline Healthy spouses & 106 & $56 / 50$ & $66(32-95)$ & - & - & - \\
\hline Neurological controls & 173 & $92 / 81$ & $67(32-88)$ & - & - & - \\
\hline \multicolumn{7}{|l|}{ Belgium } \\
\hline SALS & 153 & $97 / 56$ & $59(18-84)$ & $33(27-39)$ & 115 & 38 \\
\hline Healthy controls & 426 & $204 / 222$ & 54 (19-95) & - & - & - \\
\hline \multicolumn{7}{|l|}{ England (Birmingham) } \\
\hline SALS & 90 & $57 / 33$ & $61(16-87)$ & $30(25-35)$ & 74 & 16 \\
\hline Healthy spouses & 96 & $26 / 70$ & $60(28-84)$ & - & - & - \\
\hline \multicolumn{7}{|l|}{ England (London) } \\
\hline SALS & 158 & $99 / 59$ & $58(26-79)$ & $38(31-46)$ & 106 & 52 \\
\hline Healthy controls & 143 & $84 / 59$ & $58(27-88)$ & - & - & - \\
\hline
\end{tabular}

aMedian age (with lowest and highest values in parentheses) at onset for individuals with ALS and at blood sampling for controls. ${ }^{b}$ Kaplan-Meier analysis was used to estimate the median (95\% c.i.) survival in months after diagnosis. ' ${ }^{C}$ Survival of deceased Swedish individuals with ALS was measured in years: individuals survived for $<4$ years (76\% of SALS $77 \%$ of FALS), $4-10$ years (20\% of SALS; $18 \%$ of FALS) or $>10$ years ( $4 \%$ of SALS; $5 \%$ of FALS).

Of the Swedish individuals with SALS and FALS, Belgian individuals with SALS and Birmingham and London individuals with SALS, $92 \%, 68 \%, 65 \%, 40 \%$ and $76 \%$, respectively, had died and $8 \%, 23 \%, 17 \%, 60 \%$ and $16 \%$, respectively, were censored at the time of study; the others were lost for analysis.

carrying the at-risk A allele had a greater risk for ALS than those carrying the $\mathrm{G}$ allele (odds ratio $(\mathrm{OR})=1.3 ; 95 \%$ confidence interval (c.i.) $=$ 1.0-1.7). When comparing genotypes at position $-1,154$, we found that $\mathrm{A} / \mathrm{A}$ but not $\mathrm{G} / \mathrm{A}$ conferred an additional risk for ALS relative to $\mathrm{G} / \mathrm{G}(\mathrm{OR}=2.4 ; P=0.006$ for $\mathrm{A} / \mathrm{A} ; \mathrm{OR}=0.9 ; P=0.76$ for $\mathrm{G} / \mathrm{A})$, indicating that the at-risk effect of the A allele was recessive. Overall, a greater fraction of affected individuals than controls was A/A homozygous ( $13 \%$ versus $6 \% ; P=0.001$; recessive model).

\section{VEGF variations in FALS and SALS in the Swedish population}

The Swedish ALS cohort consisted of 292 individuals with SALS and 57 with FALS without mutations in SOD1 (Table 1). To assess whether the observed association was true for FALS as well as for SALS, we stratified our sample accordingly. The $-1,154 \mathrm{~A}$ allele frequency was similar in individuals with FALS and with SALS ( 0.35 and 0.33 , respectively) and was higher in both than in the controls (0.28). When comparing genotypes at position $-1,154$, only A/A conferred

Table 2 Association of variations in VEGF and individuals with ALS in Sweden

\begin{tabular}{|c|c|c|c|c|c|c|}
\hline \multirow[b]{2}{*}{ Genotype } & \multirow{2}{*}{$\begin{array}{c}\text { Controls } \\
\text { Number } \\
\text { (frequency; } n=275 \text { ) }\end{array}$} & \multicolumn{5}{|c|}{ Individuals with ALS } \\
\hline & & $\begin{array}{c}\text { Number } \\
\text { (frequency; } n=349 \text { ) }\end{array}$ & $P$ value & $\begin{array}{l}\text { Crude OR } \\
\text { (95\% c.i.) }\end{array}$ & $\begin{array}{l}\text { Logistic OR } \\
(95 \% \text { c.i.) }\end{array}$ & $P$ value $^{a}$ \\
\hline$-2,578 \mathrm{C} / \mathrm{C}$ & $70(0.25)$ & $88(0.25)$ & & & & \\
\hline$-2,578 \mathrm{C} / \mathrm{A}$ & $148(0.54)$ & $173(0.50)$ & & $0.9(0.6-1.4)$ & $0.9(0.6-1.4)$ & 0.72 \\
\hline$-2,578 \mathrm{~A} / \mathrm{A}$ & $57(0.21)$ & $88(0.25)$ & $0.39^{b}$ & $1.2(0.8-1.9)$ & $1.2(0.7-1.9)$ & 0.50 \\
\hline$-2,578 \mathrm{C}$ & $288(0.52)$ & $349(0.50)$ & & & & \\
\hline$-2,578 \mathrm{~A}$ & $262(0.48)$ & $349(0.50)$ & $0.41^{c}$ & $1.1(0.9-1.4)$ & & \\
\hline$-1,154 \mathrm{G} / \mathrm{G}$ & $138(0.50)$ & $162(0.46)$ & & & & \\
\hline$-1,154 \mathrm{G} / \mathrm{A}$ & $122(0.44)$ & $141(0.40)$ & & $1.0(0.7-1.4)$ & $0.9(0.7-1.3)$ & 0.76 \\
\hline$-1,154 \mathrm{~A} / \mathrm{A}$ & $15(0.06)$ & $46(0.13)$ & $0.001^{d}$ & $2.6(1.4-4.9)$ & $2.4(1.3-4.6)$ & 0.006 \\
\hline$-1,154 \mathrm{G}$ & $398(0.72)$ & $465(0.67)$ & & & & \\
\hline$-1,154 \mathrm{~A}$ & $152(0.28)$ & $233(0.33)$ & $0.029^{c}$ & $1.3(1.0-1.7)$ & & \\
\hline$-634 \mathrm{G} / \mathrm{G}$ & $145(0.53)$ & $179(0.51)$ & & $0.9(0.5-1.5)$ & $0.9(0.7-1.3)$ & 0.72 \\
\hline$-634 \mathrm{G} / \mathrm{C}$ & $108(0.39)$ & $138(0.40)$ & & $0.9(0.5-1.6)$ & $0.8(0.4-1.5)$ & 0.44 \\
\hline$-634 \mathrm{C} / \mathrm{C}$ & $22(0.08)$ & $32(0.09)$ & $0.86^{b}$ & & & \\
\hline$-634 G$ & $398(0.72)$ & $496(0.71)$ & & $0.9(0.7-1.2)$ & & \\
\hline$-634 C$ & $152(0.28)$ & $202(0.29)$ & $0.61^{c}$ & & & \\
\hline
\end{tabular}

${ }^{a} P$ values of ORs (logistic regression) were calculated after adjustment for gender and age. ${ }^{b} P$ values were calculated using the $\chi^{2}$ contingency test with 2 d.f. ${ }^{c} P$ values were calculated using the $\chi^{2}$ contingency test with 1 d.f. ${ }^{d}$ Calculation of ORs for the $-1,154 \mathrm{G} \rightarrow \mathrm{A}$ SNP indicated a recessive model. Therefore the $P$ value under the assumption of a recessive model is given.

Distribution of genotypes (frequencies) for the indicated SNPs in individuals with ALS and controls. ORs were calculated relative to genotypes $-2,578 C / C,-1,154 \mathrm{G} / \mathrm{G}$ or $-634 \mathrm{C} / \mathrm{C}$ or alleles $-2,578 \mathrm{C},-1,154 \mathrm{G}$ or $-634 \mathrm{C}$. 
Table 3 Standardized pairwise maximum-likelihood linkage disequilibria ( $\left.D^{\prime}\right)$ for individuals with SALS and controls

\begin{tabular}{lcccccccc}
\hline & \multicolumn{2}{c}{ Belgium } & \multicolumn{2}{c}{ Sweden } & \multicolumn{2}{c}{ Birmingham } & London \\
\hline & $-1,154 G / A$ & $-634 G / C$ & $-1,154 G / A$ & $-634 G / C$ & $-1,154 G / A$ & $-634 G / C$ & $-1,154 G / A$ & $-634 G / C$ \\
$-2,578 C / A$ & $0.999 / 0.935$ & $-0.999 /-0.969$ & $0.974 / 0.999$ & $-0.940 /-0.944$ & $0.966 / 0.999$ & $-0.999 /-0.950$ & $0.978 / 0.955$ & $-0.999 /-0.856$ \\
$-1,154 G / A$ & & $-0.999 /-0.968$ & & $-0.999 /-0.999$ & $-0.999 /-0.999$ & $-0.999 /-0.913$ \\
\hline
\end{tabular}

an additional risk for FALS relative to $\mathrm{G} / \mathrm{G}(\mathrm{OR}=3.4 ; P=0.005)$ and for SALS $(\mathrm{OR}=2.5 ; P=0.006)$. Individuals homozygous with respect to the $-1,154 \mathrm{~A}$ allele were less prevalent among controls $(6 \%)$ than among individuals with FALS or SALS $(18 \%, P=0.001$, and $12 \%, P=$ 0.004 , respectively; recessive model). To extend our findings, we included two additional control populations with similar age and gender distribution and drawn from the same geographic and ethnic region. The first population, consisting of 173 individuals admitted to the hospital for neurological disorders distinct from ALS, served as neurological controls to examine whether the A allele was specifically associated with ALS and not with any other neurological disorder. The second population consisted of 106 spouses of individuals with ALS. The observed $-1,154 \mathrm{~A}$ allele frequency in the populations was 0.27 and 0.26 , respectively, similar to that in the initial control population. In the remaining analysis, we focused our attention on the population with SALS, because its pathogenesis is unknown and because only individuals with SALS were available in populations from other areas that we examined (see below).

\section{VEGF haplotypes and SALS in the Swedish population}

We also determined haplotypes, because susceptibility to complex diseases is often attributable to multiple alleles whose collective effects may be more readily predicted by determining the full haplotype information. Pairwise maximum-likelihood analysis of linkage disequilibrium $\left(\mathrm{D}^{\prime}\right)$ showed a high degree of linkage disequilibrium among the SNPs (Table 3). We then inferred haplotypes at positions $-2,578,-1,154$ and -634 by the maximum-likelihood method and compared their frequencies in individuals with SALS versus controls (Supplementary Table 1 online). Only four of the eight possible three-loci haplotypes occurred at an appreciable frequency. Of those, haplotype $-2,578 \mathrm{~A} /-1,154 \mathrm{~A} /-634 \mathrm{G}$ (hereafter called haplotype AAG) was more prevalent in affected individuals than controls ( 0.33 versus 0.26; $P=0.006$ ), indicating that it is the at-risk haplotype for the Swedish population. We confirmed the four common haplotypes using independent methods (Supplementary Table 2 online). When we translated genotypes into haplotype combinations, we found that only individuals who were homozygous with respect to haplotype AAG (AAG/AAG) had significantly greater risk for SALS (OR = 2.0; c.i. $=1.2-3.2 ; P=0.007)$. Swedish individuals who were homozygous with respect to haplotype $-2,578 \mathrm{~A} /-1,154 \mathrm{G} /-634 \mathrm{G}$ (AGG/AGG) also had greater risk $(\mathrm{OR}=1.3)$. This difference did not reach significance because of insufficient power but became significant in the metaanalysis (see below).

\section{Low VEGF plasma levels in at-risk individuals with ALS}

The analysis in Vegfa ${ }^{\partial / \partial}$ mice indicated that low levels of Vegfa cause motoneuron degeneration ${ }^{17}$. VEGF plasma levels were also $\sim 50 \%$ lower in individuals with SALS (median value $39 \mathrm{pg} \mathrm{m}^{-1} ; n=195$ ) than in unaffected spouses $\left(71 \mathrm{pg} \mathrm{ml}^{-1} ; n=105 ; P<0.0001\right.$ after adjustment for age and gender). VEGF plasma levels did not correlate with age at onset, with bulbar versus spinal onset or with progression of disease. Within each group (individuals with SALS and unaffected spouses), VEGF plasma levels were lowest in individuals carrying the at-risk AAG/AAG or AGG/AGG genotypes, but VEGF levels were always lower in affected individuals than in controls of the same genotype (Fig. 1b). VEGF levels were also lowest in Belgian individuals carrying the AAG/AAG or AGG/AGG genotypes (see below). Thus, low VEGF plasma levels are associated with the genotypes conferring the greatest risk for ALS. The small sample size of individuals with FALS who were homozygous with respect to the AAG and AGG haplotypes $(n=6)$ precluded meaningful correlation of VEGF plasma levels with these at-risk genotypes.

\section{Low VEGF expression by at-risk alleles}

To confirm that the at-risk alleles did not merely statistically associate with but instead functionally affected VEGF expression, we analyzed whether the at-risk alleles reduced VEGF gene expression in vitro. Given the strong linkage disequilibrium between the three documented SNPs, we examined their effect in a haplotype context. We cloned a luciferase reporter gene cassette downstream of the VEGF promoter and 5' UTR containing either the at-risk haplotype AAG or AGG or a control haplotype CGC (the counterpart of the AAG haplotype) and used it to transfect glioma cells, which we then exposed to normoxia or hypoxia. Univariate analysis showed significant differences between the three different constructs $(P<0.001)$. Pairwise comparison showed that the luciferase activity levels in normoxia were $41 \%$ lower for haplotype AAG $(P=0.002)$ and $30 \%$ lower for haplotype AGG $(P=0.01)$ relative to haplotype CGC. In hypoxia, luciferase activities were $35 \%$ lower for haplotype AAG $(P<0.001)$ and $43 \%$ lower for haplotype AGG $(P<0.001)$. There were no significant differences between the AAG and AGG constructs $(P=0.78)$. Thus, the at-risk SNPs, in a haplotype context, lower VEGF expression in vitro.

To examine in more detail how each of the individual SNPs influenced VEGF gene expression, we evaluated their effect separately. We were unable to assess the transcriptional activity of the $-2,578 \mathrm{C} \rightarrow \mathrm{A}$ variation, because an isolated $-2,469$ to $-2,714$ nucleotide promoter fragment did not enhance the expression of a CMV promoterluciferase reporter construct above background levels (data not shown). This finding does not exclude the possibility that the $-2,578 \mathrm{C} \rightarrow \mathrm{A}$ variation influences $V E G F$ expression in the context of the entire promoter. To assess the $-1,154 \mathrm{G} \rightarrow \mathrm{A}$ variation, we cloned a fragment of 185 nucleotides in front of the luciferase gene. Compared with the $-1,154 \mathrm{G}$ allele, luciferase expression levels by the proximal VEGF promoter containing the $-1,154 \mathrm{~A}$ allele were $25 \%$ lower in normoxia $(P<0.001)$ and $26 \%$ lower in hypoxia $(P<0.001)$.

The $-634 \mathrm{G} \rightarrow \mathrm{C}$ variation is located in the $5^{\prime}$ UTR, which is known to regulate VEGF expression at the posttranscriptional level ${ }^{21,22}$. Because little is known as to how deregulated posttranscriptional control may contribute to disease phenotypes, however, we assessed whether the at-risk $-634 \mathrm{G}$ allele affected the activity of internal ribosomal entry site B (IRES-B). This element is involved in enhancing translation initiation of $V E G F$ at the classical $\mathrm{AUG}^{21}$ and regulates the production of a large VEGF (L-VEGF) isoform, the translation of 
which is initiated at an alternative CUG1 $\operatorname{codon}^{22}$ (Fig. 1a). Analysis of the secondary structure, predicted by the mfold 2.3 folding procedure $^{23}$, of the entire $5^{\prime}$ UTR leader sequence indicated that the change $-634 \mathrm{G} \rightarrow \mathrm{C}$, for comparable stabilities $\left(\mathrm{dG}=-468 \mathrm{kCal} \mathrm{mol}^{-1}\right)$, did not affect the main part of the leader structure but substantially remodeled the region upstream of the CUG1, which is the core region of the IRES-B activity (Fig. 1c,d). To evaluate the translational activity of IRES-B, we transfected glioma cells with a bicistronic reporter plasmid. Compared with the $-634 \mathrm{C}$ allele, relative IRES-B activity with the $-634 \mathrm{G}$ allele was $16 \%$ lower in normoxia $(P=0.025)$ and $23 \%$ lower in hypoxia $(P<0.001)$. The difference was more pronounced in hypoxia because IRES-B-driven gene expression is increased under hypoxic conditions by the $-634 \mathrm{C}$ allele $(P=0.024)$ but not by the $-634 \mathrm{G}$ allele $(P=0.28)$. We determined the production of L-VEGF by transfection of an expression cassette containing the entire 5' UTR (including the CUG1 start codon of L-VEGF at -540) in-frame with the cDNA encoding the matrix-attached VEGF ${ }^{189}$ isoform $^{22}$ (Fig. 1e). Densitometric quantification of the 43-kDa L-VEGF bands in cell extracts after immunoblotting showed that, relative to the $-634 \mathrm{C}$ allele, production of L-VEGF with the $-634 \mathrm{G}$ allele was $20 \%$ lower in normoxia $(P<0.001$; Fig. 1e) and also $20 \%$ lower in hypoxia $(P=0.03)$. Because $\mathrm{L}-\mathrm{VEGF}$ is cleaved to form $23-\mathrm{kDa}$ VEGF $^{22}$, levels of the latter were also $20 \%$ lower with the $-634 \mathrm{G}$ allele $(P=0.004)$, providing further confirmation of the functional involvement of this allele.

\section{VEGF and SALS in additional populations}

In an attempt to replicate the association of low VEGF-producing genotypes with SALS in other populations, we genotyped individuals with SALS of Belgian and British origin (Table 1). We analyzed two British populations, one from Birmingham and the other from the greater London area, and matched control individuals and those with SALS for age, ethnic and geographic distributions. Genotype frequencies of the $-2,578 \mathrm{C} \rightarrow \mathrm{A},-1,154 \mathrm{G} \rightarrow \mathrm{A}$ and $-634 \mathrm{G} \rightarrow \mathrm{C}$ variations in all controls were in full accordance with Hardy-Weinberg equilibrium (Table 4). In the Belgian population, more affected individuals than controls carried the $-2,578 \mathrm{~A} / \mathrm{A}$ genotype (26\% versus $20 \% ; P=0.042$; recessive model; Table 4 ), and the $-1,154 \mathrm{~A} / \mathrm{A}$ genotype was also more prevalent in affected individuals (10\% versus $8 \%$; $P=0.24$; recessive model). The $-634 \mathrm{G}$ allele was more prevalent in affected individuals than controls $(0.69$ versus $0.61 ; P=0.009)$, and individuals who were homozygous with respect to haplotype AGG had a 5.1 times greater risk for SALS ( $P=0.006$; Table 5). In the Birmingham population, more individuals with SALS than controls carried the $-2,578 \mathrm{~A} / \mathrm{A}$ genotype (30\% versus $19 \% ; P=0.024$; recessive model) and the $-1,154 \mathrm{~A} / \mathrm{A}$ genotype ( $19 \%$ versus $6 \% ; P=0.005$; recessive model). The $-634 \mathrm{G}$ allele was also more prevalent in affected individuals than controls $(0.73$ versus $0.62 ; P=0.013)$, and individuals carrying the AAG/AAG genotype had a 3.5 times greater risk for ALS ( $P=0.006$; Table 5). In the greater London population, none of the alleles was individually associated with SALS, but individuals who were homozygous with respect to haplotype AGG had a 1.7 times greater risk (Table 5). Thus, at the genotype level, we found an association for the $-1,154 \mathrm{~A}$ allele and for the $-1,154 \mathrm{~A} / \mathrm{A}$ genotype in multiple populations (significantly in the Swedish and Birmingham populations but insignificantly in the Belgian population) and for the $-634 \mathrm{G}$ allele in the Belgian population. At the haplotype level, individuals carrying the AAG/AAG genotype had greater risk for ALS in the Swedish and British (Birmingham) populations, whereas individuals with the AGG/AGG genotype had greater risk for ALS in the Belgian and British (London) populations (though the difference in this latter population was not significant).

Unfortunately, no blood samples were available from the Belgian and British individuals with ALS to correlate VEGF levels with atrisk genotypes for ALS. Nonetheless, in an effort to replicate our observation that at-risk genotypes were associated with low VEGF

Table 4 Association of variations in VEGF and individuals with SALS

\begin{tabular}{|c|c|c|c|c|c|c|c|c|c|}
\hline \multirow[b]{3}{*}{ Genotype } & \multicolumn{3}{|c|}{ Belgium } & \multicolumn{3}{|c|}{ England (Birmingham) } & \multicolumn{3}{|c|}{ England (London) } \\
\hline & \multirow{2}{*}{$\begin{array}{c}\text { Control } \\
\text { Number } \\
\text { (frequency; } \\
n=426 \text { ) }\end{array}$} & \multicolumn{2}{|c|}{ SALS } & \multirow{2}{*}{$\begin{array}{c}\text { Control } \\
\text { Number } \\
\text { (frequency; } \\
n=96 \text { ) }\end{array}$} & \multicolumn{2}{|c|}{ SALS } & \multirow{2}{*}{$\begin{array}{c}\text { Control } \\
\text { Number } \\
\text { (frequency; } \\
n=143 \text { ) }\end{array}$} & \multicolumn{2}{|c|}{ SALS } \\
\hline & & $\begin{array}{c}\text { Number } \\
\text { (frequency; } \\
n=153 \text { ) }\end{array}$ & $P$ value & & $\begin{array}{c}\text { Number } \\
\text { (frequency; } \\
n=90 \text { ) }\end{array}$ & $P$ value & & $\begin{array}{c}\text { Number } \\
\text { (frequency; } \\
n=158 \text { ) }\end{array}$ & $P$ value \\
\hline$-2,578 \mathrm{C} / \mathrm{C}$ & $127(0.30)$ & $34(0.22)$ & & $20(0.20)$ & $21(0.23)$ & & $43(0.30)$ & $48(0.30)$ & \\
\hline$-2,578 \mathrm{C} / \mathrm{A}$ & $216(0.51)$ & $79(0.52)$ & & $57(0.59)$ & $42(0.47)$ & & $66(0.46)$ & $68(0.43)$ & \\
\hline$-2,578 \mathrm{~A} / \mathrm{A}$ & $83(0.20)$ & $40(0.26)$ & $0.042^{\mathrm{a}}$ & $19(0.19)$ & $27(0.30)$ & $0.024^{a}$ & $34(0.24)$ & $42(0.27)$ & $0.29^{a}$ \\
\hline$-2,578 \mathrm{C}$ & $470(0.55)$ & $147(0.48)$ & & $97(0.51)$ & $84(0.47)$ & & $152(0.53)$ & $164(0.52)$ & \\
\hline$-2,578 \mathrm{~A}$ & $382(0.45)$ & $159(0.52)$ & $0.016^{a}$ & $95(0.49)$ & $96(0.53)$ & $0.23^{a}$ & $134(0.47)$ & $152(0.48)$ & $0.34^{a}$ \\
\hline$-1,154 \mathrm{G} / \mathrm{G}$ & $205(0.48)$ & $68(0.44)$ & & $42(0.44)$ & $36(0.40)$ & & $66(0.46)$ & $77(0.49)$ & \\
\hline$-1,154 \mathrm{G} / \mathrm{A}$ & $187(0.44)$ & $70(0.46)$ & & $48(0.50)$ & $37(0.41)$ & & $58(0.41)$ & $64(0.41)$ & \\
\hline$-1,154 \mathrm{~A} / \mathrm{A}$ & $34(0.08)$ & $15(0.10)$ & $0.24^{a}$ & $6(0.06)$ & $17(0.19)$ & $0.005^{a}$ & $19(0.13)$ & $17(0.11)$ & $0.50^{a}$ \\
\hline$-1,154 \mathrm{G}$ & $597(0.70)$ & $206(0.67)$ & & $132(0.69)$ & $109(0.61)$ & & $190(0.66)$ & $218(0.69)$ & \\
\hline$-1,154 \mathrm{~A}$ & $255(0.30)$ & $100(0.33)$ & $0.19^{a}$ & $60(0.31)$ & 71 (0.39) & $0.049^{a}$ & $96(0.34)$ & $98(0.31)$ & $0.50^{a}$ \\
\hline$-634 G / G$ & $162(0.38)$ & $71(0.46)$ & & $34(0.35)$ & $48(0.53)$ & & $67(0.47)$ & $76(0.48)$ & \\
\hline$-634 \mathrm{G} / \mathrm{C}$ & $199(0.47)$ & $69(0.45)$ & & $51(0.53)$ & $35(0.39)$ & & $60(0.42)$ & $68(0.43)$ & \\
\hline$-634 \mathrm{C} / \mathrm{C}$ & $65(0.15)$ & $13(0.09)$ & $0.027^{b}$ & $11(0.11)$ & $7(0.08)$ & $0.024^{b}$ & $16(0.11)$ & $14(0.09)$ & $0.40^{b}$ \\
\hline$-634 \mathrm{G}$ & $523(0.61)$ & 211 (0.69) & & $119(0.62)$ & $131(0.73)$ & & $194(0.68)$ & $220(0.70)$ & \\
\hline$-634 C$ & $329(0.39)$ & $95(0.31)$ & $0.009^{a}$ & $73(0.38)$ & $49(0.27)$ & $0.013^{a}$ & $82(0.32)$ & $96(0.30)$ & $0.43^{a}$ \\
\hline
\end{tabular}

${ }^{a} P$ values were calculated using the $\chi^{2}$ contingency test with 1 d.f. for alleles and genotypes under a recessive model. ${ }^{b} P$ values were calculated using the $\chi^{2}$ contingency test with 2 d.f. 
expression in the Swedish population, we quantified VEGF serum levels in 295 control individuals from Belgium. Similar to the Swedish population, VEGF levels were lowest in individuals who were homozygous with respect to haplotypes AAG and AGG, and we observed the most significant association when comparing genotypes AAG/AAG and AGG/AGG together, relative to all other genotypes $(P=0.0005$; Fig. 1f $)$.

\section{At-risk genotypes in pooled study populations}

It is not uncommon for the same haplotype to be associated with different disease susceptibility in distinct populations. Pooling distinct study populations has, therefore, been proposed to provide a better estimate of the overall $\mathrm{risk}^{24}$. We pooled the genotypes of affected and control individuals for meta-analysis and assessed the risk of SALS after adjusting for the population of origin by logistic regression. The overall risk for SALS was 1.4 times greater with the $-2,578 \mathrm{~A} /$ A genotype (c.i. $=1.1-1.8 ; P=0.006$; recessive model), 1.5 times greater with the $-1,154 \mathrm{~A} / \mathrm{A}$ genotype (c.i. $=1.1-2.0 ; P=0.012$; recessive model) and 1.2 times greater with the $-634 \mathrm{G} / \mathrm{G}$ genotype (c.i. $=1.0-1.5 ; P=0.034$; recessive model). We also evaluated the risk for individuals who were homo- or heterozygous with respect to the various haplotypes in the meta-analysis, after assessing that a single overall logistic regression of their distribution, when accounting simultaneously for the country of origin, was significant $(P=0.046)$.
The overall risk for SALS was 1.6 times greater with genotype AAG/AAG (c.i. $=1.2-2.7 ; P=0.002$; Table 5) and 1.8 times greater with genotype AGG/AGG (c.i. $=1.0-3.3 ; P=0.045$; Table 5). The marginal significance of the AGG/AGG genotype is due to the fact that only few individuals carried this genotype. The AGG/AGG genotype is biologically relevant, however, as it was associated with low VEGF levels in both the Belgian and Swedish populations (Fig. $\mathbf{1 b}, \mathbf{f})$. The association of the at-risk genotypes with ALS is specific, as individuals who were homo- or heterozygous with respect to other haplotypes had no significantly greater risk for ALS, nor were their VEGF blood levels significantly lower.

When assessing the total risk associated with VEGF variations (AAG/AAG and AGG/AGG together), we found significant associations for the Swedish individuals with ALS $(\mathrm{OR}=1.8$; c.i. = 1.2-2.7; $P=0.006)$ as well as the individuals with SALS from Belgium (OR $=1.8 ; P=0.018)$ and Birmingham $(\mathrm{OR}=3.0 ; P=0.011)$, but there was no association for the individuals with SALS from London (OR $=1.1 ; P=0.44)$. To determine the net increase in the total number of individuals with ALS resulting from the influence of the VEGF at-risk genotypes in many individuals, we calculated the population attributable risk for genotypes AAG/AAG and AGG/AGG together. This value was $5.7 \%$ in the Swedish individuals with ALS $($ relative risk $(\mathrm{RR})=1.7$; frequency $(\mathrm{f})=0.094) 5.6 \%$ in the Belgian individuals with SALS $(\mathrm{RR}=1.7 ; \mathrm{f}=0.085)$ and $10.4 \%$ in the

Table 5 Distribution of haplotypes defined by the $-2,578,-1,154$ and -634 SNPs

\begin{tabular}{|c|c|c|c|c|c|c|c|c|c|c|c|c|c|}
\hline \multirow[t]{2}{*}{ Haplotype } & \multicolumn{3}{|c|}{ Sweden } & \multicolumn{3}{|c|}{ Belgium } & \multicolumn{3}{|c|}{ Birmingham } & \multicolumn{3}{|c|}{ London } & \multirow{2}{*}{$\begin{array}{c}\text { Pooled } \\
\text { OR (c.i.), } \\
P \text { value }\end{array}$} \\
\hline & $\begin{array}{c}\text { Controls } \\
(n=554)\end{array}$ & $\begin{array}{c}\text { SALS } \\
(n=292)\end{array}$ & $\begin{array}{l}\text { OR (c.i.) } \\
\quad P \text { value }\end{array}$ & $\begin{array}{c}\text { Controls } \\
(n=426)\end{array}$ & $\begin{array}{c}\text { SALS } \\
(n=153)\end{array}$ & $\begin{array}{c}\text { OR (c.i.) } \\
P \text { value }\end{array}$ & $\begin{array}{l}\text { Controls } \\
(n=96)\end{array}$ & $\begin{array}{c}\text { SALS } \\
(n=90)\end{array}$ & $\begin{array}{c}\text { OR (c.i.) } \\
P \text { value }\end{array}$ & $\begin{array}{c}\text { Controls } \\
(n=143)\end{array}$ & $\begin{array}{c}\text { SALS } \\
(n=158)\end{array}$ & $\begin{array}{c}\text { OR (c.i.) } \\
P \text { value }\end{array}$ & \\
\hline CGG/CGG & 0.05 & 0.04 & $\begin{array}{c}0.8 \\
(0.4-1.6)\end{array}$ & 0.02 & 0.03 & $\begin{array}{c}1.4 \\
(0.5-4.2)\end{array}$ & 0.02 & 0.03 & $\begin{array}{c}1.6 \\
(0.3-9.9)\end{array}$ & 0.05 & 0.04 & $\begin{array}{c}0.8 \\
(0.2-2.2)\end{array}$ & $\begin{array}{c}0.8 \\
(0.6-1.5) \\
0.94\end{array}$ \\
\hline CGG/CGC & 0.16 & 0.11 & $\begin{array}{c}0.7 \\
(0.4-1.1)\end{array}$ & 0.12 & 0.11 & $\begin{array}{c}0.9 \\
(0.5-1.6)\end{array}$ & 0.07 & 0.12 & $\begin{array}{c}1.8 \\
(0.7-4.8)\end{array}$ & 0.14 & 0.18 & $\begin{array}{c}1.3 \\
(0.6-2.5)\end{array}$ & $\begin{array}{c}0.9 \\
(0.7-1.2) \\
0.47\end{array}$ \\
\hline CGG/AGG & 0.10 & 0.09 & $\begin{array}{c}0.9 \\
(0.6-1.5)\end{array}$ & 0.05 & 0.06 & $\begin{array}{c}1.1 \\
(0.5-2.4)\end{array}$ & 0.08 & 0.08 & $\begin{array}{c}0.9 \\
(0.3-2.7)\end{array}$ & 0.07 & 0.07 & $\begin{array}{c}1.0 \\
(0.4-2.4)\end{array}$ & $\begin{array}{c}1.0 \\
(0.7-1.4) \\
0.90\end{array}$ \\
\hline CGG/AAG & 0.14 & 0.13 & $\begin{array}{c}0.9 \\
(0.6-1.4)\end{array}$ & 0.11 & 0.11 & $\begin{array}{c}1.1 \\
(0.6-1.9)\end{array}$ & 0.07 & 0.11 & $\begin{array}{c}1.6 \\
(0.6-4.4)\end{array}$ & 0.13 & 0.10 & $\begin{array}{c}0.8 \\
(0.4-1.6)\end{array}$ & $\begin{array}{c}1.0 \\
(0.7-1.3) \\
0.91\end{array}$ \\
\hline CGC/AGG & 0.11 & 0.10 & $\begin{array}{c}0.9 \\
(0.5-1.4)\end{array}$ & 0.12 & 0.12 & $\begin{array}{c}1.0 \\
(0.6-1.7)\end{array}$ & 0.14 & 0.09 & $\begin{array}{c}0.6 \\
(0.2-1.6)\end{array}$ & 0.07 & 0.08 & $\begin{array}{c}1.1 \\
(0.5-2.6)\end{array}$ & $\begin{array}{c}0.9 \\
(0.7-1.2) \\
0.49\end{array}$ \\
\hline CGC/AAG & 0.15 & 0.19 & $\begin{array}{c}1.3 \\
(0.9-1.9)\end{array}$ & 0.23 & 0.23 & $\begin{array}{c}1.0 \\
(0.7-1.6)\end{array}$ & 0.32 & 0.18 & $\begin{array}{c}0.5 \\
(0.2-0.9)\end{array}$ & 0.19 & 0.18 & $\begin{array}{c}0.9 \\
(0.5-1.7)\end{array}$ & $\begin{array}{c}1.0 \\
(0.8-1.3) \\
0.99\end{array}$ \\
\hline CGC/CGC & 0.07 & 0.08 & $\begin{array}{c}1.2 \\
(0.7-2.0)\end{array}$ & 0.15 & 0.08 & $\begin{array}{c}0.5 \\
(0.3-1.0)\end{array}$ & 0.11 & 0.08 & $\begin{array}{c}0.7 \\
(0.3-2.0)\end{array}$ & 0.11 & 0.09 & $\begin{array}{c}0.8 \\
(0.4-1.8)\end{array}$ & $\begin{array}{c}0.8 \\
(0.6-1.1) \\
0.19\end{array}$ \\
\hline AAG/AGG & 0.10 & 0.08 & $\begin{array}{c}0.8 \\
(0.5-1.3)\end{array}$ & 0.11 & 0.12 & $\begin{array}{c}1.1 \\
(0.6-2.0)\end{array}$ & 0.11 & 0.11 & $\begin{array}{c}1.1 \\
(0.4-2.7)\end{array}$ & 0.09 & 0.13 & $\begin{array}{c}1.6 \\
(0.7-3.4)\end{array}$ & $\begin{array}{c}1.0 \\
(0.7-1.4) \\
0.92\end{array}$ \\
\hline AGG/AGG & 0.03 & 0.04 & $\begin{array}{c}1.3 \\
(0.6-2.9) \\
0.49\end{array}$ & 0.01 & 0.05 & $\begin{array}{c}5.1 \\
0.006^{*}\end{array}$ & 0.01 & 0 & $\begin{array}{l}- \\
-\end{array}$ & 0.02 & 0.04 & $\begin{array}{c}1.8 \\
0.20^{*}\end{array}$ & $\begin{array}{c}1.8 \\
(1.0-3.3) \\
0.045\end{array}$ \\
\hline AAG/AAG & 0.07 & 0.12 & $\begin{array}{c}2.0 \\
(1.2-3.2) \\
0.007\end{array}$ & 0.08 & 0.10 & $\begin{array}{c}1.3 \\
0.19 *\end{array}$ & 0.06 & 0.19 & $\begin{array}{c}3.5 \\
0.006^{*}\end{array}$ & 0.11 & 0.10 & $\begin{array}{l}0.9 \\
0.5^{*}\end{array}$ & $\begin{array}{c}1.6 \\
(1.2-2.3), \\
0.002\end{array}$ \\
\hline
\end{tabular}

"The one-tailed P value calculated in the direction of the AAG/AAG and AGG/AGG at-risk genotypes. In the individual populations, P values are only shown for AGG/AGG and AAG/AAG genotypes, as these were significantly associated with ALS in the single overall logistic regression.

Results for rare genotypes are not shown. The AGG/AGG genotype was not detected in the British SALS population. 
Birmingham individuals with SALS $(\mathrm{RR}=2.6 ; \mathrm{f}=0.073)$. We found that the association was most significant when all populations were pooled, with a 1.7 times greater risk for ALS (c.i. $=1.3-2.2 ; P=$ 0.0002 after adjusting for the country of each population). Notably, we obtained an even more statistically significant association (OR $=1.8 ; P=0.00004)$ by calculating the Mantel-Haenszel common odds ratio (Breslow-Day homogeneity test, $P=0.32$; Fig. 1g). Logistic regression analysis setting genotypes AAG/AAG and AGG/AGG as the dependent variable, after adjusting for the country of origin, indicated that these at-risk genotypes conferred a risk for ALS in the pooled populations, independently of the subtype of disease (typical ALS or progressive bulbar paresis), age, gender or survival after diagnosis (data not shown).

\section{Low levels of Vegfa and ALS in SOD1 ${ }^{\mathrm{G} 93 \mathrm{~A}}$ mice}

The genetic association and gene expression results indicate that low VEGF-producing alleles are associated with greater risk for ALS. But, for this association to be relevant in the pathogenesis of ALS, VEGF should also have a biological effect on motoneurons. Our previous work in Vegfa $\partial / \partial$ mice suggested such a functional involvement ${ }^{17}$, and here we sought additional evidence that Vegfa stimulated motoneuron survival in two stress scenarios. First, we analyzed whether low levels of Vegfa modified motoneuron degeneration in an established ALS mouse model, and second, we evaluated whether delivery of Vegfa could prevent motoneuron death in ischemic conditions, as ischemia might be a mechanism of motoneuron degeneration ${ }^{17}$. To confirm that reduced Vegfa expression could modify the severity of motoneuron degeneration, we intercrossed mice expressing a hemizygous $S O D 1^{\mathrm{G} 93 \mathrm{~A}}$ transgene (an established mouse model of $\mathrm{ALS}^{25}$ ) with Vegfa ${ }^{\partial / \partial}$ mice expressing reduced levels of Vegfa owing to a targeted deletion of the HRE ${ }^{17}$. We mated the resultant SOD $1^{\mathrm{G} 93 \mathrm{~A}} \mathrm{Vegfa} \mathrm{a}^{\mathrm{\partial}++}$ mice to obtain $S O D 1^{\mathrm{G} 93 \mathrm{~A}} \mathrm{Vegfa}{ }^{\mathrm{\partial} / \partial}$ and SOD $1^{\mathrm{G} 93 \mathrm{~A}} \mathrm{Vegfa}{ }^{+/+}$ littermates with matched genetic backgrounds. SOD $1^{\mathrm{G} 93 \mathrm{~A}}$ mice developed severe muscle weakness due to motoneuron loss beyond 3 months of age, were able to stay for $2 \mathrm{~min}$ on a rotarod until $118 \pm 4 \mathrm{~d}$ of age and died after $124 \pm 4 \mathrm{~d}$, whereas Vegfa ${ }^{\partial / \partial}$ mice developed the first signs of muscle weakness and motoneuron loss after 5 months of age and survived for more than a year ${ }^{17}$. Compared with SOD1 ${ }^{\mathrm{G} 93 \mathrm{~A}} \mathrm{Vegfa}^{+/+}$littermates, SOD1 ${ }^{\mathrm{G} 93 \mathrm{~A}}$ Vegfa ${ }^{\text {д/d }}$ mice had significantly more muscle weakness, performed significantly worse in the rotarod test (they were unable to stay for $2 \mathrm{~min}$ on the rotarod beyond $98 \pm 2 \mathrm{~d} ; n=8 ; P=0.0001)$ and died earlier (107 $\pm 3 \mathrm{~d}$; $n=10$; $P=0.001$; Fig. 2a). A more complete description of the
SOD1 ${ }^{\mathrm{G} 93 \mathrm{~A}}$ Vegfa ${ }^{\mathrm{\partial} / \partial}$ phenotype will be reported elsewhere. These findings indicate that a promoter mutation, which reduces Vegfa expression in mice, is capable of modifying the motoneuron degeneration phenotype in an ALS mouse model.

\section{Paralysis after spinal ischemia in Vegfa ${ }^{\partial / \partial}$ mice}

If VEGF functionally affects motoneurons, levels of VEGF might be expected to regulate motoneuron survival in conditions of stress. Because ischemia might contribute to motoneuron degeneration ${ }^{17}$, we challenged $V e g f a a^{\partial / \partial}$ mice (having lower Vegfa levels) with spinal cord ischemia reperfusion at 7 weeks of age, when their motor function was

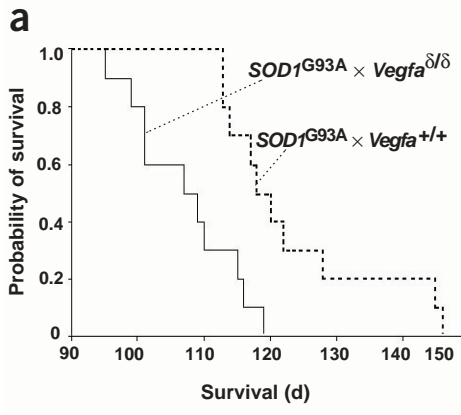

d
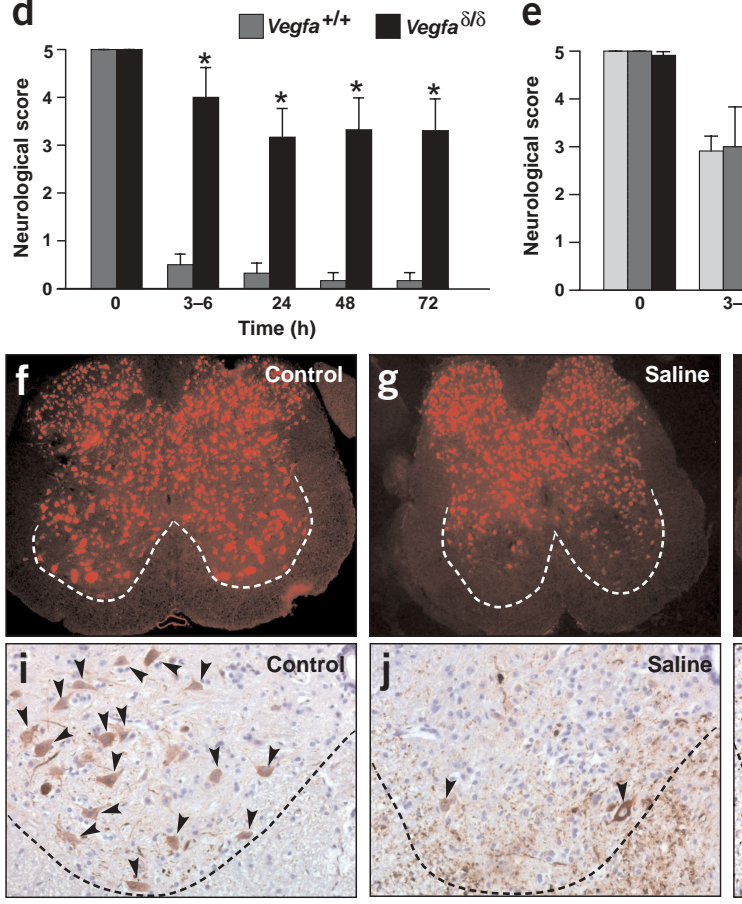

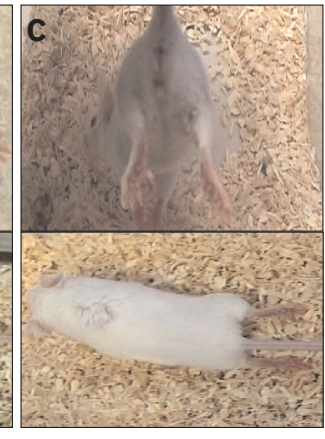

e

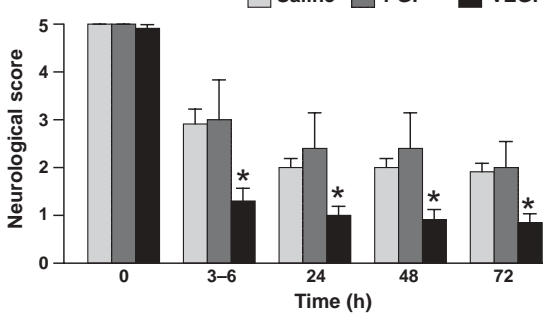

Figure 2 Role and therapeutic potential of VEGF in stimulating motoneuron survival. (a) Kaplan-Meier survival curve showing the shorter survival of SOD $1^{\mathrm{G} 93 \mathrm{~A}} \mathrm{Vegfa} \mathrm{\partial}^{\mathrm{d} / \mathrm{a}}$ mice relative to SOD $1^{\mathrm{G} 93 \mathrm{~A}} \mathrm{Vegfa}^{+/+}$ littermates $(P=0.001)$. (b,c) Illustration of the neurological outcome after acute spinal cord ischemia. (b) Mouse with score 1: hind limbs stretched and rigid when lifted by tail (upper panel), normal walking (lower panel). (c) Mouse with score 4: hind limbs immobile when lifted by tail (upper panel), unable to walk (lower panel). (d) Minimal spinal cord ischemia, which caused only transient motor impairment in wild-type mice, caused persistent paralysis in Vegfa ${ }^{\partial / \partial}$ mice. ${ }^{*} P<0.05$. (e) Administration of VEGF, but not PGF, to wild-type mice improves motor recovery after severe spinal cord ischemia. ${ }^{*} P<0.05$. (f-k) Immunostaining of motoneurons in the anterior horn at the lumbar level for NeuN (f-h) or SMI32 (i-k) from control mice (f,i) or mice after spinal cord ischemia $(\mathbf{g}, \mathbf{h}, \mathbf{j}, \mathbf{k})$. There were more immunoreactive neurons (arrowheads) after delivery of VEGF (h,k) compared with saline (g,j). SMI32 stains nonphosphorylated neurofilaments, which are normally detected in the soma (gray matter) but become detectable in the axons (white matter) on cellular stress. The border between gray and white matter is delineated with an interrupted line. 
still normal and no disease symptoms were yet observed ${ }^{17}$. We scored motor function in the hind limbs $0,6,24,48$ and $72 \mathrm{~h}$ after reperfusion using a rating scale of 0 (normal function) to 5 (absence of movement; Fig. 2b,c). To assess whether Vegfa $\partial / \partial$ mice might be more sensitive to ischemia reperfusion, we clamped the aortic arch, left subclavian artery and internal mammary artery for only $8 \mathrm{~min}$, as this insult caused only transient paralysis in wild-type mice (Fig. 2b,d). Only one of six wild-type mice had a neurological score of 1 after $3 \mathrm{~d}$. In contrast, all six Vegfa $a^{\partial / \partial}$ mice were severely paralyzed for the entire duration of the analysis $(3 \mathrm{~d})$ and they did not recover neurologically $(n=$ 6 ; $P=0.0004$; Fig. 2 c,d). Thus, a relatively minor ischemic insult that caused only a transient clinical deficit in wild-type mice caused persistent paralysis in Vegfa $\partial / \partial$ mice because of their reduced levels of Vegfa.

\section{Administration of VEGF protects mice against motoneuron loss} Encouraged by the genetic evidence in humans and mice that Vegfa levels functionally determine motoneuron survival, we analyzed whether delivery of VEGF to wild-type mice might salvage motoneurons at risk of irreversible damage after spinal cord ischemia reperfusion, a model previously used to evaluate the therapeutic potential of riluzole, a drug currently used to treat $\mathrm{ALS}^{26,27}$. Because clamping the thoracic vessels for only $8 \mathrm{~min}$ resulted in a transient paralysis with almost complete recovery after $24 \mathrm{~h}$, we developed a more severe model of motoneuron loss to evaluate the therapeutic potential of VEGF. When we prolonged the clamping duration to $12 \mathrm{~min}$, all saline-treated wild-type mice were severely paralyzed and only partially recovered after $3 \mathrm{~d}$ (Fig. 2e). Daily intraperitoneal administration of $2 \mu \mathrm{g}$ VEGF significantly protected wild-type mice against permanent paralysis. Despite a similar degree of paralysis immediately after clamping, the neurologic outcome of VEGF-treated mice improved more rapidly and extensively $(n=12 ; P=0.0004$; Fig. 2 e). The effect of VEGF was specific, as delivery of the VEGF homolog PGF (previously called PLGF), which binds a different receptor and does not affect motoneuron survival ${ }^{17}$, was ineffective $(n=5 ; P=$ not significant; Fig. 2e). Histopathology showed that there was severe loss of motoneurons in the lumbar gray matter of saline-treated mice but milder injury in VEGF-treated mice. Of the $126 \pm 11$ NeuN-positive neurons normally present per ventral horn section in unligated mice, $47 \pm 5 \%$ were lost after spinal cord ischemia in saline-treated mice, but only $32 \pm 3 \%$ were lost in VEGF-treated mice $(n=9 ; P<0.05$; Fig. $\mathbf{2} \mathbf{f}-\mathbf{h})$. Staining of non-phosphorylated neurofilaments using the SMI32 antibodies showed an even more significant protection by VEGF; of the $23 \pm 2$ SMI32-positive neurons normally present per ventral horn section in unligated mice, only $20 \pm 3 \%$ remained after spinal cord ischemia in saline-treated mice, but up to $37 \pm 4 \%$ were still present in VEGF-treated mice ( $n=9 ; P=0.01$; Fig. $2 \mathbf{i}-\mathbf{k})$. NeuNand SMI32-positive neurons in the ventral horn at the thoracic level were comparable in all groups of mice (data not shown). Thus, whereas reduced VEGF levels predispose to motoneuron loss in mice and humans, delivery of VEGF protects against loss of at-risk motoneurons after stress.

\section{DISCUSSION}

The pathogenesis of SALS is largely unknown ${ }^{1-4,7,10,15}$. Even though VEGF does not map to previously identified genomic regions linked to $\mathrm{ALS}^{6,7}$, the genetic findings in Vegfa $\partial / \partial$ mice, in which we modified the HRE in the VEGF promoter $^{17}$, raised the question of whether $V E G F$ might act in the genetic pathway of motoneuron degeneration in humans. Because we detected no sequence variations in the HRE sequence of over 400 individuals with ALS from different European countries, we examined whether variations of SNPs in VEGF might confer greater risk for ALS. In particular, we focused on the SNPs $-2,578 \mathrm{C} \rightarrow \mathrm{A},-1,154 \mathrm{G} \rightarrow \mathrm{A}$ and $-634 \mathrm{G} \rightarrow \mathrm{C}$, as they were previously documented to correlate with downregulated VEGF expression ${ }^{19,20}$. Genotyping populations of affected individuals and controls from Sweden, Belgium and England showed that these individual SNPs had strong linkage disequilibrium and, as is often the case, interacted with each other to determine, in combination, susceptibility to ALS. Of all possible combinations, haplotypes $-2,578 \mathrm{~A} /-1,154 \mathrm{~A} /-634 \mathrm{G}$ (AAG) and $-2,578 \mathrm{~A} /-1,154 \mathrm{G} /-634 \mathrm{G}$ (AGG) were significantly associated with greater risk for ALS. Even though the risk associated with the atrisk genotypes AAG/AAG and AGG/AGG differed in the individual populations, the overall risk for ALS was consistently 1.8-3.0 times greater when analyzing both at-risk genotypes together. Only in the greater London population did we not find an association. When assessing the risk in a meta-analysis of all populations and after adjusting for the population of origin, however, genotypes AAG/AAG and AGG/AGG together had a 1.8 times greater risk $(P=$ $0.00004)$. Calculation of the population-attributable risk indicated that the influence of the VEGF at-risk genotypes resulted in a net increase in the total number of individuals with ALS by 5.7, 5.6 and $10.4 \%$ in the Swedish, Belgian and Birmingham populations, respectively. Although this may seem a modest effect, it is relevant when comparing it to the role of mutations in SOD1, which are responsible for $1-2 \%$ of the entire ALS population ${ }^{1-4,7,10,15}$. Defining the attributable risk of VEGF genotypes in ALS subpopulations might provide further insights into pathogenesis and therapeutic interventions.

Only $5-10 \%$ of all individuals with ALS have a family history and of those only $14 \%$ (France) to $23 \%$ (US and Scandinavia) are attributable to mutations in SOD1 (refs. 6,7). It is thus noteworthy that we found a similar association of VEGF genotypes in individuals with FALS and with SALS who have no mutations in SOD1, as the pathogenesis of FALS is probably heterogeneous. It indicates that VEGF has a relevant role in modifying motoneuron degeneration, even when inherited disease genes determine the latter. Because of the limited availability of individuals with FALS with mutations in $S O D 1$, we could not analyze whether variations in VEGF would also modify the disease characteristics in individuals with FALS carrying mutations in SOD1. But mice carrying mutations in both SOD1 (ref. $25)$ and the Vegfa promoter (SOD $1^{\mathrm{G} 93 \mathrm{~A}}$ Vegfa ${ }^{\partial / \partial}$ mice) died earlier because of more severe motoneuron degeneration. These genetic data in mice thus underscore the need for evaluating whether VEGF also modifies motoneuron degeneration in individuals with FALS and mutations in SOD1.

Although each of the at-risk alleles that we identified has previously been correlated with lower VEGF levels in vivo or in vitro $^{19,20,28}$, we cannot exclude the possibility that they are in linkage disequilibrium with additional (perhaps more functionally relevant) SNPs. We found, however, that VEGF plasma levels were most significantly reduced in individuals with ALS and in healthy individuals with the at-risk genotypes AAG/AAG or AGG/AGG and that these at-risk genotypes reduced expression of VEGF in vitro. Moreover, our findings indicate that the at-risk $-1,154 \mathrm{~A}$ allele reduces $V E G F$ gene transcription, and the $-634 \mathrm{G}$ allele impairs IRES-dependent translation of the normally secreted VEGF isoforms, thus suggesting a molecular explanation for the previously documented lower expression of VEGF by cells or subjects carrying these alleles ${ }^{19,20,28}$. The unusually long 5' UTR of VEGF contains two internal ribosome entry sites (IRES-A at nucleotide -294 to -1 and IRES-B at nucleotide -948 to -556 ) involved in the enhancement of translation 
of AUG-initiated VEGF ${ }^{21}$. Analysis of the predicted secondary structure of the $5^{\prime}$ UTR indicated that the secondary structure of the IRES-B sequence was significantly remodeled for the common $-634 \mathrm{G}$ or variant $-634 \mathrm{C}$ allele. Because an optimal secondary structure is essential for normal IRES function, a possible explanation for the impaired IRES-dependent VEGF translation by the $-634 \mathrm{G}$ allele is that the IRES-B secondary structure of this allele was less optimal than that of the $-634 \mathrm{C}$ allele. Precedent for diseases caused by mutation of the IRES function is exemplified by a mutation in the nervespecific connexin 32 causing the Charcot-Marie-Tooth disease ${ }^{29}$. An interesting question emerging from these data is whether the haplotypes are important for greater risk of ALS or whether this effect is instead determined by the individual alleles. Although the SNP genotypes themselves were sufficient to obtain a statistically significant association in the meta-analysis, we found a more consistent and significant association at the haplotype level. Another unexplained question is why the risk of ALS was not greater in heterozygous AAG/AGG individuals and why their reduced circulating VEGF levels were not as low as those of the homozygous at-risk individuals.

We observed that the $-634 \mathrm{G}$ allele impaired production of the LVEGF isoform. L-VEGF is 205 amino acid residues longer than the AUG-initiated VEGF forms because translation is initiated at CUG1 (nt -540 in the 5' UTR; ref. 22). Analysis of the secondary structure of the $5^{\prime}$ UTR showed that this initiation codon is located in the immediate vicinity of $-634 \mathrm{G} \rightarrow \mathrm{C}$ and could therefore be influenced by variations of this polymorphic site. L-VEGF is proteolytically cleaved at the peptide signal sequence and converted to the shorter isoforms (VEGF ${ }^{121}$, VEGF $^{165}$ and VEGF ${ }^{189}$ isoforms; Fig. 1a; ref. 22), which are secreted into the extracellular milieu and known to have biological effects. Although the role of L-VEGF is not clear, it may serve as a reservoir for generation of the shorter isoforms ${ }^{21}$. It is therefore tempting to speculate that insufficient production of the LVEGF by the $-634 \mathrm{G}$ allele might have contributed to the reduced $V E G F$ expression levels, which we identified in the present study as a risk factor of ALS. If true, this would provide the first insight in a pathophysiological role of the L-VEGF isoform in disease. Support for such a hypothesis is provided by previous evidence that N-terminally extended isoforms of bFGF or c-myc, the translation of which is also initiated in the $5^{\prime}$ UTR, have been implicated in medically important disorders ${ }^{30-32}$.

We could not document any sequence variation in the HRE of the VEGF promoter. Because HIF1A and EPAS1 bind the HRE, mutation in these genes could also affect expression of VEGF. But we did not detect any functionally relevant missense mutations in HIF1A and EPAS1. How can we then reconcile the mouse and human genetic data? Notably, we previously documented that baseline VEGF expression levels in Vegfa ${ }^{\partial / \partial}$ mice were reduced by $\sim 25 \%$, presumably because the HRE deletion also interfered with additional transcriptional regulators ${ }^{17}$. We therefore speculate that variant alleles resulting in reduced VEGF expression levels in baseline normoxic conditions as well as in response to hypoxia might lead to motoneuron degeneration. The lack of functional variations in the HRE or in HIF1A and EPAS1 does not exclude the possibility that impaired hypoxic regulation of VEGF might still contribute to greater ALS risk. In fact, the hypoxia-induced increase in IRES-B activity of the -634C but not of the $-634 \mathrm{G}$ allele supports such a hypothesis. Considering the complexity of the transcriptional and translational regulation of $V E G F$ expression ${ }^{33}$, additional SNPs might also be involved in determining the susceptibility to ALS.

Genetic association studies are often plagued by irreproducibility, particularly when a single, small population ( $<150$ subjects $)$ is studied $^{24,34}$. We therefore did meta-analysis on a large population and replicated the association in geographically different populations. Our analysis in almost 2,000 individuals is, to the best of our knowledge, the largest genetic association study with ALS, a disease that is less common than complex traits, such as diabetes or hypertension. After analyzing four different populations, we found a significant association of the low VEGF-producing haplotypes with ALS in 1,668 individuals from three geographically distinct regions (Swedish, Belgian, British/Birmingham) but no association in the 301 individuals from the British/London study group. Such inconsistency in replication is a well recognized phenomenon in genetic association studies ${ }^{24,34}$. Although we do not know the precise reason for the lack of association in the London study, it does not necessarily negate the positive association in the other populations or minimize the relevance of the overall association on meta-analysis of the pooled populations ${ }^{35}$. It is also worth noting that $V E G F$ is not an obligatory disease-associated gene like SOD1, mutations in which cause ALS regardless of the genetic background or environmental influences. Instead, VEGF is a modifier of ALS whose influence may depend on genetic background, additional modifiers, environment or lifestyle. It is well known that VEGF levels are influenced by environmental changes. Future analysis of how VEGF genetically interacts with previously proposed modifier genes, such as PSEN1 (ref. 13), SOD2 (ref. 36), $C_{N T F^{37}}$, $A P O E^{16}, L I F^{38}$, or SMN2 (ref. 14), in determining the overall disease susceptibility might also be helpful.

The genetic association of low VEGF-producing haplotypes with ALS in humans was not the result of a random genome-wide scan but instead was prompted by and now replicates our previous mouse genetic findings that low Vegfa levels contribute to greater susceptibility to motoneuron death ${ }^{17}$. Our findings that Vegfa $\partial / \partial$ mice are more sensitive to a relatively minor ischemic insult, which causes only a transient clinical deficit in normal mice, underscores the conclusion that VEGF levels determine the susceptibility to motoneuron disease. Moreover, the more severe motoneuron death in SOD1 ${ }^{\mathrm{G} 93 \mathrm{~A}} \mathrm{Vegfa} \mathrm{g}^{\mathrm{\partial} / \mathrm{\partial}}$ mice, the protective effect of VEGF against ischemic motoneuron death and the low plasma levels of VEGF in individuals with ALS relative to spouses all support a functional role of VEGF, as suggested by the human genetic association. A function for VEGF in neuronal survival, regeneration, growth, differentiation and axonal outgrowth is also implied by a growing body of recent publications ${ }^{39-47}$. Although our VEGF treatment data only relate to acute spinal cord ischemia, our findings may bear some relevance to ALS, as chronic ischemia might contribute to motoneuron death ${ }^{17}$. The findings also raise the intriguing question whether more long-term treatment with VEGF might delay the onset or slow the progression of adult-onset motoneuron degeneration as well.

\section{METHODS}

Study populations and sample collection. We analyzed populations from Sweden, Belgium and England (Table 1). None of the individuals with SALS had a documented familial history of ALS to the first degree of relatedness.

Swedish population. Individuals with ALS were unrelated citizens who reported (northern) Swedish citizenship for at least three generations and were referred to the Umea University ALS Clinic. Only individuals with SALS or FALS without mutations in SOD1 were included. For the Swedish control samples, we used 275 individuals from the Betula prospective cohort study of Umea, Sweden ${ }^{48}$. To match the Betula controls to the individuals with ALS as a group, we sampled controls from age cohorts of 50, 55, 60, 65 and 70 years. Potential control subjects with neurodegenerative disorders were excluded. In all instances, we contacted spouses of individuals with ALS, and a total of 106 
spouses agreed to take part in the study. Individuals who immigrated to Sweden during any of the last three generations and their descendants were excluded. We applied similar criteria to select neurological control samples from individuals admitted to the Department of Neurology, Umea University Hospital for non-ALS related neurological disorders (headache, conversion syndrome, epilepsy, hydrocephalus, stroke, multiple sclerosis, multifocal motor neuropathy, etc.).

Belgian population. The Belgian population of individuals with ALS consisted of 153 unrelated subjects of Flemish origin who were referred to the University Hospital of Leuven for SALS ${ }^{49}$. The Flemish control population consisted of healthy spouses of individuals with dementia and randomly sampled healthy individuals. The control population was matched as a group to have a similar age, geographical and ethnical distribution as the affected individuals. All individuals in the Belgian sample self-reported Flemish ethnicity for at least three generations.

British (Birmingham and London) population. Individuals with SALS were British citizens with British surnames, admitted to the Department of Neurology, School of Medicine in Birmingham or to King's College in London. No details were available about the ancestry of the previous generations, but the included subjects were probably of English, Welsh, Scottish and Irish ancestry. Healthy controls were matched as a group to have similar age, ethnicity and geographic distribution. The Birmingham controls consisted of spouses of individuals with ALS and, if unavailable, spouses of individuals with Parkinson disease. The London controls were healthy controls sampled from the hospital staff.

Clinical data. We obtained the following clinical data from affected individuals: gender, age at onset of disease and duration of disease (or survival; categorized into three classes: $<4$ years, $4-10$ years and $>10$ years for the Swedish population; in months for the Belgian and British populations). We categorized the type of disease at onset as (i) typical ALS with onset in the legs, arms, chest or abdominal muscles and clinical evidence for lesions to both the upper motoneuron systems in the brain and the lower motoneuron systems in the spinal cord or (ii) progressive bulbar paresis, also called ALS with bulbar onset, a type of motoneuron degeneration with onset in the muscles in the face, mouth and throat. We analyzed the clinical data blindly before the results of the genetic studies were available. Ethical approval for this study was granted by the Ethics Committee from each of the participating institutions and informed consent was obtained from all subjects.

DNA extraction. We extracted DNA from white blood cells from peripheral blood using the QIAamp Blood Kit (Qiagen).

DHPLC analysis. We screened 250 Swedish and 96 Belgian individuals for SNPs in the HRE by denaturing high-performance liquid chromatography (DHPLC) analysis, using a Transgenomic WAVE instrument. We also screened 96 Swedish individuals with SALS and 200 controls for variations in the exons and exon/intron boundaries of HIF1A and EPAS1. We designed primers using the Primer3 software program. We loaded crude PCR products on a DNASep column and eluted them from the column using an acetonitrile gradient in a $0.1 \mathrm{M}$ triethylamine acetate buffer, $\mathrm{pH} 7$, at a constant flow rate of $0.9 \mathrm{ml} \mathrm{min}{ }^{-1}$. The gradient was created by mixing eluent $\mathrm{A}$ (0.1 M triethylamine acetate, $0.1 \mathrm{M} \mathrm{Na}_{4}$ EDTA) and eluent B (25\% acetonitrile in $0.1 \mathrm{M}$ triethylamine acetate). The gradient and temperature required for successful resolution of heteroduplex molecules were predicted by Wavemaker version 3.4.4. Primer sequences and PCR and sequencing conditions are available on request.

DNA sequencing. We used direct sequencing (Big Dye terminator cycle sequencing kit, Applied Biosystems) of PCR fragments with aberrant DHPLC patterns to confirm the presence of a variant allele.

Pyrosequencing. We genotyped SNPs in the promoter and 5' UTR by pyrosequencing. We immobilized biotinylated PCR products on streptavidin-coated paramagnetic beads (Dynal). We obtained biotinylated single-stranded DNA by incubating the immobilized PCR products in $50 \mu \mathrm{l} 0.5 \mathrm{M} \mathrm{NaOH}$ for $5 \mathrm{~min}$ and then washing twice in $100 \mu 110 \mathrm{mM}$ Tris-Acetate, $\mathrm{pH}$ 7.6. We carried out primer annealing by incubation at $80^{\circ} \mathrm{C}$ for $2 \mathrm{~min}$ and then at room temperature for $5 \mathrm{~min}$. We carried out pyrosequencing on the PSQ96 pyrosequencer (Pyrosequencing). To assess population substructure, we pyrosequenced five biallelic SNPs (on chromosome 2, 5, 7, 17 and 18) for the four control populations. In addition, we genotyped a panel of five tetranucleotide STR markers $(5,10,12,15,21)$. Primer sequences and PCR and sequencing conditions are available on request.

VEGF levels. To determine plasma VEGF levels, we collected blood in 10-ml vacuum tubes containing EDTA (Becton Dickinson Vacutainer Systems Europe), quickly centrifuged it and immediately stored plasma fractions at $-80^{\circ} \mathrm{C}$ until analysis. To determine VEGF concentrations in serum, we drew blood into serum tubes containing clot activator (Becton Dickinson Vacutainer Systems Europe) and allowed it to coagulate for at least $30 \mathrm{~min}$ at room temperature before centrifugation. We subdivided samples into aliquots and stored them at $-30{ }^{\circ} \mathrm{C}$ until analysis. We drew samples during morning office hours. We used the commercial Quantikine human VEGF ELISA kit (R\&D System) to measure plasma and serum concentrations of VEGF.

Statistical analysis. Common or variant alleles were defined as the allele with the highest or lowest frequency, respectively. Haplotypes were considered rare when their frequency was $1 \%$ or less. Deviations from Hardy-Weinberg equilibrium were tested using the observed allele frequency by the $\chi^{2}$ contingency test ( 1 degree of freedom, d.f.). We assessed differences in distribution of alleles and genotypes between the groups by calculating the Pearson $\chi^{2}$, from which we derived $P$ values with the appropriate d.f. number ( 2 d.f. for genotypes, 1 d.f. for alleles or genotypes under a recessive model). As the descriptive measure of association between genotypes and alleles, we calculated crude ORs, their 95\% c.i. ranges and corresponding $P$ values relative to the common (for the $-1,154 \mathrm{G} \rightarrow \mathrm{A}$ and $-2,578 \mathrm{C} \rightarrow \mathrm{A}$ SNP) or variant (for $-634 \mathrm{G} \rightarrow \mathrm{C} \mathrm{SNP}$ ) genotype or allele. To adjust ORs for age and gender, we carried out logistic regression using the statistical software program SPSS version 10.0, yielding similar levels of association and statistical significance. We used the maximum-likelihood method to estimate haplotype frequencies $\left(\mathrm{EH}\right.$ program ${ }^{50}$ ) to determine estimates of disequilibrium $\left(D_{i j}\right)$ between pairwise combinations of alleles (here $D_{i j}=h_{i j}-p_{i} q_{j}$ and $p_{i}$ and $q_{j}$ are the frequencies for allele $i$ at locus 1 and for allele $j$ at locus 2). We calculated the linkage disequilibrium coefficient $D_{i j}^{\prime}$ standardized by its maximum value as $\mathrm{D}^{\prime}=\mathrm{D} / \mathrm{D}_{\max }$. If $\mathrm{D}<0$, then $\mathrm{D}_{\max }=-\mathrm{pq}$; if $\mathrm{D}>0$, then $\mathrm{D}_{\max }=\mathrm{p}(1-\mathrm{q})$. We analyzed differences in frequencies of particular genotypes (hetero- or homozygosity with respect to haplotypes) between affected individuals and controls by calculating logistic odds ratios of the at-risk genotypes relative to the other genotypes as a group. When analyzing pooled populations, we pooled individual genotypes of affected individuals and controls and carried out logistic regression to adjust for the population of origin. We tested the specific hypothesis, suggested by our previously published transgenic mouse data ${ }^{17}$, that reduced VEGF levels might increase the risk for motoneuron degeneration by genotyping in the Swedish population those SNPs in the promoter and $5^{\prime}$ UTR of VEGF predicted to reduce VEGF expression ${ }^{19,20}$. Then, to replicate our findings, we compared individuals with SALS in two other populations (Belgian and British) with their randomly ascertained local controls. For the Swedish population and all pooled analyses, we calculated two-tailed $P$ values. In replication populations (British and Belgian), we calculated one-tailed $P$ values for all alleles in the direction of their appearance in the at-risk haplotype AAG. Because we knew in advance the direction of the test in the replication studies and wanted to test only the influence of the at-risk genotypes, we used one-tailed probabilities. We obtained one-sided type I probabilities for the $\chi^{2}$ test by halving the two-sided probabilities calculated by SPSS. For ORs, there were no confidence intervals when one-tailed $P$ values were calculated. Results were considered statistically significant when their type I probability was less than 0.05 . We did not carry out a Bonferroni correction for several reasons: (i) we tested a specific hypothesis that was suggested by previously obtained biological data ${ }^{17}$; (ii) this hypothesis was sustained by the replication studies and extended by the correlation of VEGF levels with the at-risk genotypes and haplotypes; (iii) the three SNPs did not segregate independently but were in significant linkage disequilibrium; and (iv) we first tested the distribution of all genotypes (hetero- and homozygosity with respect to haplotypes) 
together in a single overall logistic regression, accounting simultaneously for the country of origin. We evaluated the at-risk genotypes in the separate subpopulations only because this logistic regression was overall significant $(P=$ 0.046 ) and because we found significant ORs for genotypes AAG/AAG and AGG/AGG (on their own relative to all other genotypes). To calculate the Mantel-Haenszel common OR of the at-risk genotypes and test for homogeneity (Breslow-Day homogeneity test) we used SPSS. The population attributable risk was estimated using the Levin formula: PAR $=100 \times$ (proportion of total population exposed $\times($ relative risk for exposed versus nonexposed -1$)) /$ ( (proportion of total population exposed $\times$ (relative risk for exposed versus nonexposed -1$))+1$ ). We calculated relative risk ratios as the ratio of at-risk frequencies between affected individuals and controls. We evaluated the distribution of the quantitative variables by the Kolmogorov-Smirnof test for Gaussian normality. When the distribution was Gaussian, we compared quantitative variables between groups by parametric analysis of variance (e.g., expression experiments). When this was not the case, such as for the VEGF levels, we used the non-parametric Kruskall-Wallis analysis of variance to compare several groups and the Mann-Whitney $U$ test to compare their central tendency measures. We used SPSS version 10 for these tests. To assess the degree of population substructure in the four control sample sets, we selected 96 Swedish (population-based), 96 Belgian and 48 Birmingham spouses and 48 London control samples and genotyped them for five SNPs and five STRs from five different chromosomes and with an allele frequency of $>0.25$ in each population. Hardy-Weinberg equilibrium was fulfilled for all SNPs and STRs in each population, except for one SNP in the Belgian sample group $(P=0.024)$, suggesting that large-scale population differences are not apparent in the samples.

Luciferase reporter assays. To test the relative ability of the various haplotypes (e.g., AAG, AGG and CGC) to modify gene expression, we fused the fragment from $-2,469$ to $-2,714$ (harboring the $-2,578 \mathrm{C} \rightarrow \mathrm{A} \mathrm{SNP}$ ) to the fragment from $-1,176$ to -405 (harboring the $-1,154 \mathrm{G} \rightarrow \mathrm{A}$ and $-634 \mathrm{G} \rightarrow \mathrm{C}$ SNPs) and cloned it into the firefly luciferase plasmid pGL3-Basic (Promega). We introduced the various $-2,578,-1,154$ or -634 alleles using the site-directed mutagenesis kit (Stratagene). We transfected reporter plasmids into human glioma GI-1 cells (RCB0763, RIKEN Cell Bank), grown in Dulbecco's modified Eagle medium with 10\% fetal bovine serum, using the FuGENE6 Transfection Reagent (Roche Diagnostics). As an internal control, we cotransfected $0.1 \mu \mathrm{g}$ of the renilla luciferase plasmid pRL-TK containing the herpes simplex virus-thymidine kinase (HSV-TK) promoter with $1 \mu \mathrm{g}$ of the firefly luciferase vector. At $16 \mathrm{~h}$ after transfection, we cultured cells for $8 \mathrm{~h}$ in normoxia or hypoxia ( $2 \%$ $\mathrm{O}_{2}$ ) and collected them for measurement of firefly and renilla luciferase activities using the Dual-Luciferase Reporter Assay System (Promega). We also evaluated the transcriptional activity of the $-2,578 \mathrm{C} \rightarrow \mathrm{A}$ SNP alone by cloning the fragment from $-2,469$ to $-2,714$ in front of the minimal CMV promoter, driving the luciferase reporter gene, in the pGL3 promoter plasmid. We similarly evaluated the transcriptional/promoter activity of the isolated $-1,154 \mathrm{G} \rightarrow \mathrm{A}$ SNP by cloning the fragment from $-1,172$ to -987 in front of the luciferase marker gene in the pGL3 basic plasmid. We introduced the various $-2,578$ or $-1,154$ alleles using the site-directed mutagenesis kit (Stratagene).

IRES-B activity. We used a bicistronic vector containing two different reporter cistrons, the renilla and firefly genes encoding the luciferase enzyme, designated LUCr and LUCf, respectively, separated by the 5' UTR of VEGF (from -947 to -484 ) in the intercistronic region ${ }^{21}$. Translation of LUCr is cap-dependent, whereas translation of LUCf is dependent on the IRES-B in the $5^{\prime}$ UTR region upstream of LUCf. The LUCf/LUCr ratio after transfection in C6 glioma cells served as a measure of the relative IRES-B activity.

L-VEGF production. We transfected COS cells with an expression cassette containing the entire $5^{\prime}$ UTR (including the CUG1 start codon of L-VEGF at $-540)$ in-frame with the cDNA encoding the matrix-attached VEGF ${ }^{189}$ isoform $^{22}$. We cloned a cassette containing the common $-634 \mathrm{G}$ or variant $-634 \mathrm{C}$ allele by site-directed mutagenesis. This L-VEGF was predicted to contain 394 amino acid residues and have a molecular weight of $43 \mathrm{kDa}$. After Western blotting using an antibody specific to L-VEGF 22 , we used densitometry to quantify the L-VEGF and VEGF ${ }^{189}$ bands. We carried out experiments at least four times in triplicate.
Mouse studies. We intercrossed mice expressing a $S O D 1^{\mathrm{G} 93 \mathrm{~A}}$ transgene (obtained from the Jackson Laboratory) with Vegfa ${ }^{\partial / \partial}$ mice expressing low levels of Vegfa owing to a targeted deletion of the $\mathrm{HRE}^{17}$. We then mated the resultant SOD $1^{\mathrm{G} 93 \mathrm{~A}}$ Vegfa ${ }^{\partial /+}$ mice to obtain $S O D 1^{\mathrm{G} 93 \mathrm{~A}} \mathrm{Vegfa}{ }^{\partial / \partial}$ and $S O D 1^{\mathrm{G} 93 \mathrm{~A}}$ $\mathrm{Vegfa}^{+/+}$littermates with matched genetic backgrounds. We scored muscle weakness using the rotarod as described ${ }^{17}$. We carried out spinal cord ischemia reperfusion by clamping the aortic arch, left subclavian artery and internal mammary artery as described ${ }^{26,27}$, except that we used normal wild-type mice with a Swiss genetic background or Vegfa ${ }^{\partial / \partial}$ mice with a mixed genetic background ${ }^{17}$. In control Swiss mice, the clamping time $\left(12 \mathrm{~min} ; 36^{\circ} \mathrm{C}\right)$ was optimized to yield a partially reversible paralysis of the hind limbs after $24-72 \mathrm{~h}$ with acceptable perioperational mortality (10-15\%), allowing us to evaluate the therapeutic potential of VEGF. In Vegfa/d mice, the thoracic vessels were only clamped for $8 \mathrm{~min}$ to obtain a transient and less severe paralysis. Motor function in the hind limbs was scored $0,1,3,6,24,48$ and $72 \mathrm{~h}$ after reperfusion using a rating scale of 0 (normal function), 1 (hind limbs are stretched but remain rigid when the mouse is lifted by its tail, normal walking), 2 (no stretching of hind limbs when lifted by tail, normal walking), 3 (rigid hind limbs when lifted by tail, ataxic or knuckling walking), 4 (rigid hind limbs when lifted by tail, hind limb movement but unable to walk) and 5 (rigid hind limbs when lifted by tail, total absence of movement). We analyzed neuropathological signs of motoneuron loss histologically as described ${ }^{17}$. We administered $2 \mu \mathrm{g}$ of VEGF ${ }^{165}$ (R\&D Systems) or PGF2 (Reliatech) intraperitoneally $1 \mathrm{~d}$ before the surgery and then daily until $2 \mathrm{~d}$ after surgery. Neurological and histological outcome was scored independently by two investigators blinded to the treatment type.

Note: Supplementary information is available on the Nature Genetics website.

\section{ACKNOWLEDGMENTS}

The authors thank A. Claeys and B. VanKeirsbilck for technical support, G. Breier for plasmids, the VIB Genetic Service Facility for pyrosequencing, B. Dermaut and C. van Duijn for critical discussions of the statistical analyses and K. Brepoels, A. Bouché, M. Demol, E. Gils, B. Hermans, S. Janssen, W. Man, A. Manderveld, K. Maris, W. Martens, C. Nijs, M. Nijs, S. Meynen, P. Van Wesemael, A. Van den Hoeck, B. Vanwetswinkel and S. Wyns for assistance. D.L. is supported by the Flemish Institute for the promotion of scientific research and E.S. by the Fund for Scientific Research, Belgium. This work is supported by grants from the Fund for Scientific Research, the Concerted Research Actions, Belgium and the European Union to P.C.; from the Scientific Projects Committee University of Birmingham to K.M.; from the Fund for Scientific Research and the Interuniversity Attraction Pole program to C.V.B. and W.R.; from Saitama Medical School Research Center and Maruki Memorial Special Scholarship to T.A.; and from the Nutrition and Toxicology and Growth and Development Research Institutes in Maastricht to R.V.

\section{COMPETING INTERESTS STATEMENT}

The authors declare that they have no competing financial interests.

Received 3 January; accepted 13 June 2003

Published online 6 July 2003; doi:10.1038/ng1211

1. Shaw, C.E., Al-Chalabi, A. \& Leigh, N. Progress in the pathogenesis of amyotrophic lateral sclerosis. Curr. Neurol. Neurosci. Rep. 1, 69-76 (2001).

2. Brown, R.H.Jr. \& Robberecht, W. Amyotrophic lateral sclerosis: pathogenesis. Semin. Neurol. 21, 131-139 (2001).

3. Rowland, L.P. \& Shneider, N.A. Amyotrophic lateral sclerosis. N. Engl. J. Med. 344, 1688-1700 (2001)

4. Cleveland, D.W. \& Rothstein, J.D. From Charcot to Lou Gehrig: deciphering selective motor neuron death in ALS. Nat. Rev. Neurosci. 2, 806-819 (2001).

5. Turner, M.R., Parton, M.J. \& Leigh, P.N. Clinical trials in ALS: an overview. Semin. Neurol. 21, 167-175 (2001).

6. Siddique, T. \& Lalani, I. Genetic aspects of amyotrophic lateral sclerosis. Adv. Neurol. 88, 21-32 (2002).

7. Andersen, P.M. Genetics of sporadic ALS. Amyotroph. Lateral. Scler. Other Motor Neuron Disord. 2 Suppl 1, S37-S41 (2001).

8. Hand, C.K. \& Rouleau, G.A. Familial amyotrophic lateral sclerosis. Muscle Nerve 25 135-159 (2002).

9. Yang, Y. et al. The gene encoding alsin, a protein with three guanine-nucleotide exchange factor domains, is mutated in a form of recessive amyotrophic lateral sclerosis. Nat. Genet. 29, 160-165 (2001).

10. Julien, J.P. Amyotrophic lateral sclerosis. unfolding the toxicity of the misfolded. Cell 104, 581-591 (2001). 
11. Figlewicz, D.A. et al. Variants of the heavy neurofilament subunit are associated with the development of amyotrophic lateral sclerosis. Hum. Mol. Genet. 3, 1757-1761 (1994).

12. Al-Chalabi, A. et al. Deletions of the heavy neurofilament subunit tail in amyotrophic lateral sclerosis. Hum. Mol. Genet. 8, 157-164 (1999).

13. Panas, M. et al. Genotyping of presenilin-1 polymorphism in amyotrophic lateral sclerosis. J. Neurol. 247, 940-942 (2000).

14. Veldink, J.H. et al. Homozygous deletion of the survival motor neuron 2 gene is a prognostic factor in sporadic ALS. Neurology 56, 749-752 (2001).

15. Siddique, T. et al. Lack of association between apolipoprotein $\mathrm{E}$ genotype and sporadic amyotrophic lateral sclerosis. Neurogenetics 1, 213-216 (1998).

16. Lacomblez, L. et al. APOE: a potential marker of disease progression in ALS Neurology 58, 1112-1114 (2002).

17. Oosthuyse, B. et al. Deletion of the hypoxia-response element in the vascular endothelial growth factor promoter causes motor neuron degeneration. Nat. Genet. 28, 131-138 (2001).

18. Skene, J.P. \& Cleveland, D.W. Hypoxia and Lou Gehrig. Nat. Genet. 28, 107-108 (2001).

19. Shahbazi, M. et al. Vascular endothelial growth factor gene polymorphisms are associated with acute renal allograft rejection. J. Am. Soc. Nephrol. 13, 260-264 (2002).

20. Awata, T. et al. A common polymorphism in the $5^{\prime}$-untranslated region of the VEGF gene is associated with diabetic retinopathy in type 2 diabetes. Diabetes $\mathbf{5 1}$ 1635-1639 (2002).

21. Huez, I. et al. Two independent internal ribosome entry sites are involved in translation initiation of vascular endothelial growth factor mRNA. Mol. Cell. Biol. 18, 6178-6190 (1998).

22. Huez, I., Bornes, S., Bresson, D., Creancier, L. \& Prats, H. New vascular endothelia growth factor isoform generated by internal ribosome entry site-driven CUG translation initiation. Mol. Endocrinol. 15, 2197-2210 (2001).

23. Mathews, D.H., Sabina, J., Zuker, M. \& Turner, D.H. Expanded sequence dependence of thermodynamic parameters improves prediction of RNA secondary structure. J. Mol. Biol. 288, 911-940 (1999).

24. Altshuler, D. et al. The common PPAR $\gamma$ Pro12Ala polymorphism is associated with decreased risk of type 2 diabetes. Nat. Genet. 26, 76-80 (2000).

25. Gurney, M.E. The use of transgenic mouse models of amyotrophic lateral sclerosis in preclinical drug studies. J. Neurol. Sci. 152 Suppl 1, S67-S73 (1997).

26. Lang-Lazdunski, L. et al. Ischemic spinal cord injury induced by aortic cross-clamping: prevention by riluzole. Eur. J. Cardiothorac. Surg. 18, 174-181 (2000).

27. Lang-Lazdunski, L. et al. Spinal cord ischemia. Development of a model in the mouse. Stroke 31, 208-213 (2000).

28. McCarron, S.L. et al. Influence of cytokine gene polymorphisms on the development of prostate cancer. Cancer Res. 62, 3369-3372 (2002).

29. Hudder, A. \& Werner, R. Analysis of a Charcot-Marie-Tooth disease mutation reveals an essential internal ribosome entry site element in the connexin-32 gene. J. Biol. Chem. 275, 34586-34591 (2000).

30. Lazaris-Karatzas, A., Montine, K.S. \& Sonenberg, N. Malignant transformation by a eukaryotic initiation factor subunit that binds to mRNA $5^{\prime}$ cap. Nature 345 544-547 (1990)

31. West, M.J., Sullivan, N.F. \& Willis, A.E. Translational upregulation of the c-myc oncogene in Bloom's syndrome cell lines. Oncogene 11, 2515-2524 (1995).
32. Prats, A.C. \& Prats, H. Translational control of gene expression: role of IRESs and consequences for cell transformation and angiogenesis. Prog. Nucleic Acid Res. Mol. Biol. 72, 367-413 (2002).

33. Ferrara, N. Role of vascular endothelial growth factor in regulation of physiological angiogenesis. Am J. Physiol. Cell Physiol. 280, C1358-C1366 (2001).

34. Ioannidis, J.P., Ntzani, E.E., Trikalinos, T.A. \& Contopoulos-loannidis, D.G. Replication validity of genetic association studies. Nat. Genet. 29, 306-309 (2001).

35. Lohmueller, K.E., Pearce, C.L., Pike, M., Lander, E.S. \& Hirschhorn, J.N. Metaanalysis of genetic association studies supports a contribution of common variants to susceptibility to common disease. Nat. Genet. 33, 177-182 (2003).

36. Landeghem, G.F., Tabatabaie, P., Beckman, L., Beckman, G. \& Andersen, P.M. MnSOD signal sequence mutation associated with sporadic motor neuron disease. Eur. J. Neurol. 6, 639-644 (1999).

37. Giess, R. et al. Early onset of severe familial amyotrophic lateral sclerosis with a SOD- 1 mutation: potential impact of CNTF as a candidate modifier gene. Am. J. Hum. Genet. 70, 1277-1286 (2002).

38. Giess, R. et al. Potential role of LIF as a modifier gene in the pathogenesis of amyotrophic lateral sclerosis. Neurology 54, 1003-1005 (2000).

39. Yang, Z.J. et al. Role of vascular endothelial growth factor in neuronal DNA damage and repair in rat brain following a transient cerebral ischemia. J. Neurosci. Res. 70 140-149 (2002).

40. Svensson, B. et al. Vascular endothelial growth factor protects cultured rat hippocampal neurons against hypoxic injury via an antiexcitotoxic, caspase-independent mechanism. J. Cereb. Blood Flow Metab. 22, 1170-1175 (2002).

41. Wick, A. et al. Neuroprotection by hypoxic preconditioning requires sequential activation of vascular endothelial growth factor receptor and Akt. J. Neurosci. 22, 6401-6407 (2002)

42. Facchiano, F. et al. Promotion of regeneration of corticospinal tract axons in rats with recombinant vascular endothelial growth factor alone and combined with adenovirus coding for this factor. J Neurosurg. 97, 161-168 (2002).

43. Matsuzaki, H. et al. Vascular endothelial growth factor rescues hippocampal neurons from glutamate-induced toxicity: signal transduction cascades. FASEB J. 15, 1218-1220 (2001).

44. Jin, K. et al. Vascular endothelial growth factor (VEGF) stimulates neurogenesis in vitro and in vivo. Proc. Natl. Acad. Sci. USA 99, 11946-11950 (2002).

45. Carmeliet, P. \& Storkebaum, E. Vascular and neuronal effects of VEGF in the nervous system: implications for neurological disorders. Semin. Cell Dev. Biol. 13, 39-53 (2002).

46. Sondell, M., Sundler, F. \& Kanje, M. Vascular endothelial growth factor is a neurotrophic factor which stimulates axonal outgrowth through the flk-1 receptor. Eur. J. Neurosci. 12, 4243-4254 (2000).

47. Li, B., Xu, W., Luo, C., Gozal, D. \& Liu, R. VEGF-induced activation of the PI3-K/Akt pathway reduces mutant SOD1-mediated motor neuron cell death. Brain Res. Mol. Brain Res. 111, 155-164 (2003).

48. Nilsson, L.G. et al. The betula prospective cohort study: memory, health and aging. Aging Neuropsychol. Cogn. 4, 1-32 (1996).

49. Thijs, V., Peeters, E., Theys, P., Matthijs, G. \& Robberecht, W. Demographic characteristics and prognosis in a Flemish amyotrophic lateral sclerosis population. Acta Neurol. Belg. 100, 84-90 (2000).

50. Terwilliger, J.D. \& Ott, J. Handbook for Human Genetic Linkage (Johns Hopkins University Press, Baltimore, 1994). 